\title{
Chronik der Medienentwicklung in Deutschland 2004
}

\author{
Christiane Matzen
}

1. Medienregulierung (Medienpolitik / Medienrecht)

2. Medienunternehmen

3. Medienmärkte

3.1 Medienangebote / Medieninhalte Presse

3.2 Medienangebote / Medieninhalte Rundfunk

3.2.1 Fernsehen

3.2.2 Radio

3.3 Medienangebote / Medieninhalte Online

3.4 Werbung / Marktanteile

4. Mediennutzung

\section{Medienregulierung (Medienpolitik / Medienrecht)}

Am 8. Januar schlägt die KEF in ihrem 14. Bericht eine Erhöhung der monatlichen Rundfunkgebühr um 1,09 Euro auf 17,24 Euro zum 1. Januar 2005 vor. ARD und ZDF hatten eine Gebührenerhöhung um ca. 12 Prozent bzw. rund 1,90 Euro für die nächste Periode von 2005-2008 beantragt. Gleichzeitig kritisiert die KEF, dass die ARD noch immer keine klaren Kriterien für eine Begrenzung ihres Engagements im Online-Bereich auf programmbezogene Inhalte vorgelegt habe, wie sie der neue, am 1. April in Kraft tretende Rundfunkstaatsvertrag fordert. Die KEF kürzte daher die von der ARD insgesamt angemeldete Summe für Internet-Aktivitäten von 207,8 Mio. Euro um 58,3 Mio. Euro (=28 \%).

Nach Ausscheiden des bisherigen Direktors der Landesrundfunkzentrale Mecklenburg-Vorpommern (LRZ) am 16. Januar kann der im Juli 2003 zum neuen Direktor gewählte Uwe Hornauer sein Amt zunächst nicht wie geplant antreten, da der stellvertretende LRZ-Direktor und Justitiar, Norbert Nordmeyer, eine Konkurrentenklage vor dem Verwaltungsgericht Schwerin eingereicht hatte. Am 5. März entscheidet das Oberverwaltungsgericht Greifswald in letzter Instanz gegen die Klage. Hornauer tritt sein Amt daraufhin am 15. März an.

Am 27. Januar veröffentlicht die ARD ein Positionspapier ${ }^{1}$ zur Debatte um eine Strukturreform des öffentlich-rechtlichen Rundfunks, die sich nach Vorschlägen der Ministerpräsidenten Stoiber, Steinbrück und Milbradt entwickelt hatte. Die ARD plädiert darin für eine Trennung von Gebührenanpassung und Reformdebatte.

Der Deutsche Werberat rügt am 27. Januar einen Spot, mit dem MTV für seinen Ableger MTV 2 Pop geworben hatte und der u. a. auf RTL 2 ausgestrahlt worden war. Der Spot stelle einen „Missbrauch menschlicher Sexualität für kommerzielle Zwecke“ dar. Die für VIVA zuständige Landesanstalt für Medien Nordrhein-Westfalen beanstandet auf ihrer Sitzung am 30. Januar die Ausstrahlung von Werbespots der Vereinten Dienstleistungsgewerkschaft (Verdi) zum Thema Ausbildungsplätze. Damit habe VIVA gegen das Verbot der Ausstrahlung von politischer Werbung verstoßen.

1 Die Zusammenfassung des Papiers ist abgedruckt in Funkkorrespondenz 5/2004 vom 30. Januar 2004, S. 30f., Anhang 2 des Papiers ist dokumentiert in epd medien, Nr. 6/2004 vom 28.1.2004, S. $28 \mathrm{ff}$. 
Das Oberlandesgericht Hamm urteilt am 4. Februar in zweiter Instanz, dass der Sender ProSieben, „TV-Total“-Moderator Stefan Raab und die beiden Produktionsfirmen Raab TV und Brainpool 70.000 Euro Schadensersatz an die 19-jährige Lisa Loch zahlen müssen, deren Persönlichkeitsrechte durch mehrere Sendungen schwer verletzt worden seien.

ARD und ZDF geben am 22. Februar bekannt, dass sie den Vertrag mit der Kabelgesellschaft Deutschland KDG zur Verbreitung der öffentlich-rechtlichen digitalen Angebote aus dem Jahr 2003 gekündigt haben. Die von der KDG geplante Vergabe von Gütesiegeln ausschließlich an Pay-TV-taugliche Endgeräte sei eine Behinderung des entstehenden Marktes für digitale Kabelempfangsgeräte. Strittig bleibe außerdem die Entwicklung der Multimedia Home Platform (MHP) in den Kabelnetzen. Außerdem sei keine Verständigung „zu einer diskriminierungsfreien Abbildung“ der öffentlich-rechtlichen frei zugänglichen Angebote in der elektronischen Programmübersicht der KDG, dem Navigator, erreicht worden. Auch die Privatsender hatten gegen die Pläne der KDG in Zusammenhang mit dem Start der digitalen Verbreitung ab 1. April protestiert.

Der Bundesverband der Deutschen Zeitungsverleger (BDZV), der Deutsche Journalistenverband (DJV) und die Deutsche Journalistenunion (dju) einigen sich am 25. Februar über einen neuen Tarifvertrag für Zeitungsredakteure. Das Urlaubsgeld soll danach auf 80 Prozent eines Monatsgehaltes gekürzt, die Urlaubsdauer nach Alter gestaffelt und das Gehalt rückwirkend ab 1. August 2003 um 1,3 Prozent erhöht werden. Die Erhöhung soll bis zum 31. Juli 2005 gelten. In den vorausgegangenen vier Wochen hatten bis zu 3000 Redakteure gestreikt.

Der Berliner Initiativkreis öffentlich-rechtlicher Rundfunk legt Anfang März eine Stellungnahme ${ }^{2}$ zur laufenden Diskussion um eine Rundfunkstrukturreform vor. Zu den rund 40 Mitgliedern des Initiativkreises zählen u. a. frühere Intendanten und Direktoren von ARD und ZDF, Hochschulprofessoren, Journalisten, Künstler sowie ehemalige Minister bzw. Senatoren von Landesregierungen. Der Kölner Initiativkreis Öffentlicher Rundfunk fordert in einer Resolution ${ }^{3}$ vom 1. November eine konstruktive Weiterentwicklung des Public-Service-Status beim Rundfunk und bezieht sich damit u. a. auf den Vortrag „Entwicklungsperspektiven der Medienpolitik in Deutschland“4 der Beauftragten der Bundesregierung für Kultur und Medien, Christina Weiss. Diese hatte sich darin am 20. September für eine auf Online-Möglichkeiten zielende Erweiterung des Amsterdamer Protokolls ausgesprochen, das den EU-Mitgliedsstaaten weitgehend die Ausgestaltung des öffentlichen Rundfunks zugesteht.

Am 19. März verabschiedet der Medienrat der Landesmedienanstalt Saarland (LMS) eine Resolution zur Medienethik im Fernsehen ${ }^{5}$. Darin kritisiert das Gremium u. a., dass bei neuen Unterhaltungssendungen wie etwa der Dschungelshow „Ich bin ein Star - holt mich hier raus!“ (RTL), „Big Brother“ (RTL 2) oder „Fear Factor“ (RTL) gesellschaftliche Normen missachtet und Geschmacksgrenzen ignoriert würden.

Der Sportchef des Hessischen Rundfunks (HR), Jürgen Emig, tritt am 23. März zurück, nachdem ihm vorgeworfen wurde, von kleineren Vereinen und Veranstaltern seit Jahren Geld für Sportübertragungen in Form von Produktionskostenzuschüssen eingefordert sowie Schleichwerbung im Fernsehen des HR unterstützt zu haben. Die Staatsanwaltschaft Frankfurt leitet im April ein Strafermittlungsverfahren wegen des

2 Abgedruckt in Funkkorrespondenz Dokumentation 16/2004 vom 13. April 2004, S. $25 \mathrm{ff}$.

3 Abgedruckt in epd medien 91/2004 vom 20. November 2004, S. $10 \mathrm{ff}$.

4 Abgedruckt in epd medien 78/2004 vom 18. Oktober 2004, S. $18 \mathrm{ff}$.

5 Abgedruckt in Funkkorrespondenz 18/2004 vom 30. April 2004, S. 31. 
Verdachts der Bestechlichkeit und wettbewerbsbeschränkender Absprachen gegen ihn sowie zwei Mitarbeiter von Marketing-Firmen ein.

Die ARD-Intendanten stimmen am 30. März dem Abschluss des 8. Film-/Fernsehabkommens mit der Filmförderungsanstalt (FFA) entsprechend dem neuen, seit Anfang 2004 in Kraft befindlichen Filmfördergesetz zu. Die ARD wird danach bis 2008 jährlich 5,5 Mio. Euro und damit doppelt so viel wie zuvor für die Unterstützung von Kino- und Fernsehfilmen in Geld- und Sachleistungen aufbringen. Da das ZDF die gleiche Verpflichtung eingeht, bringt der öffentlich-rechtliche Rundfunk insgesamt 11 Mio. Euro auf. Am 15. Oktober 2003 hatte VPRT-Präsident Jürgen Doetz anlässlich einer Anhörung des Ausschusses für Kultur und Medien des Deutschen Bundestages angekündigt, dass auch die privaten Fernsehveranstalter ab 2004 das Volumen ihrer Filmförderung verdoppeln werden, u. a. durch vermehrt kostenlos zur Verfügung gestellte Promotionspots.

Auf der gleichen Hauptversammlung einigen sich die ARD-Intendanten mit den Vorsitzenden der Rundfunk- und Verwaltungsräte der Sender auf „Richtlinien für die Zusammenarbeit im ARD-Gemeinschaftsprogramm ,Erstes Deutsches Fernsehen' und anderen Gemeinschaftsprogrammen und -angeboten“6. Mitte September legen ARD, ZDF und das Deutschlandradio ihre nach dem neuen Rundfunkstaatsvertrag geforderten „Selbstverpflichtungen für die künftige Programmgestaltung“ vor, die alle zwei Jahre überarbeitet werden sollen. Der Hörfunkrat des Deutschlandradios hatte seinen „Bericht über programmliche Leistungen und Perspektiven des Deutschlandradios 2004-2006“7 am 9. September verabschiedet, das ZDF beschloss seine 21-seitigen „Programmperspektiven des ZDF 2004-2006“8 am 13. September und die ARD-Hauptversammlung folgte mit der Verabschiedung der 19-seitigen „Leitlinien für die Programmgestaltung der ARD für die Jahre 2005/2006“9 einen Tag später. In allen drei Selbstverpflichtungserklärungen bekennen sich die Sender zu einem verantwortungsbewussten, qualitätsvollen Programm, in dem Information und Kultur eine entscheidende Rolle spielen.

Am 1. April tritt der 7. Rundfunkänderungsstaatsvertrag ${ }^{10}$ in Kraft. Darin wird der Funktionsauftrag des öffentlich-rechtlichen Rundfunks konkretisiert. In einer Protokollerklärung, die auf Initiative Bayerns hinzugefügt wird, werden die Hörfunkveranstalter, vor allem die öffentlich-rechtlichen, aufgefordert, deutschsprachige Musik stärker zu berücksichtigen und durch entsprechende Sendeplätze zu fördern. ${ }^{11}$

Die EU-Kommission richtet am 12. April zwei Auskunftsersuchen zum öffentlichrechtlichen Rundfunk an die Bundesregierung Deutschlands. Ein Auskunftsersuchen bezieht sich vorrangig auf behauptete staatliche Beihilfen zu Gunsten von Film- und Fernsehproduktionstöchtern, die direkt oder mittelbar im Besitz von ARD-Anstalten

6 Abgedruckt in Funkkorrespondenz Dokumentation 16/2004 vom 13. April 2004, S. 21ff., und in epd medien, 25/2004 vom 3. April 2004, S. $25 \mathrm{ff}$.

7 Abgedruckt in epd medien 79/2004 vom 9. Oktober 2004, S. $26 \mathrm{ff}$.

8 Abgedruckt in epd medien 79/2004 vom 9. Oktober 2004, S. $15 \mathrm{ff}$.

9 Abgedruckt in epd medien 79/2004 vom 9. Oktober 2004, S. $3 \mathrm{ff}$.

10 Abgedruckt in Funkkorrespondenz Dokumentation 20/2004 vom 10. Mai 2004 und epd medien 76/2003 vom 27. September 2003, S. $32 \mathrm{ff}$.

11 Der Anteil von Musikneuheiten liegt bei den öffentlich-rechtlichen Radioprogrammen bei $14,3 \%$, darunter sind 1,2 \% deutschsprachig, bei den Privaten beträgt der Anteil von Musikneuheiten 17,1 \%, davon 0,6 \% in deutscher Sprache; vgl. Funkkorrespondenz 28/2003 vom 11. Juli 2003, S. 21. 
sind (Bavaria, Studio Hamburg, Drefa); ${ }^{12}$ private deutsche Produktionsfirmen hatten hierzu Beschwerde eingereicht. Im zweiten Auskunftsersuchen geht es generell um vermutete staatliche Beihilfe für öffentlich-rechtliche Rundfunkanstalten, ${ }^{13}$ wie sie der Verband Privater Rundfunk und Telekommunikation (VPRT) als Beschwerdeführer behauptet hatte. Ein besonderer Abschnitt fragt nach der 2001 begonnenen Zusammenarbeit zwischen dem ZDF und der T-Online International AG beim Online-Angebot www.heute.t-online.de. Die EU-Wettbewerbsdirektion will prüfen, ob das ZDF im Rahmen dieser Partnerschaft unter normalen Marktbedingungen gehandelt und in der Zusammenarbeit Rundfunkgebühren verwendet oder aber „Gewinne erzielt hat, die bei der Ermittlung des Finanzbedarfs der Rundfunkanstalt in Abzug gebracht wurden“. Die Behörde der Bundesbeauftragten für Kultur und Medien erarbeitet mit den Bundesländern bis 15. Juli eine mit den betroffenen Rundfunkanstalten ARD und ZDF abgestimmte Antwort.

Das Ermittlungsverfahren gegen den Glücksspielsender 9Live wegen Betrugs bei Telefongewinnspielen wird am 23. April mangels Tatverdacht eingestellt. Es sei nicht nachzuweisen, so die Staatsanwaltschaft München, dass der Fernsehsender technisch in der Lage sei, bei den Telefonanrufen von Zuschauern zu manipulieren. Gegen 9Live hatten insgesamt 186 Zuschauer Strafanzeige erstattet, weil der Sender für Anrufe Telefongebühren berechnet haben soll, obwohl ein Besetzzeichen zu hören gewesen sei.

RTL und Sat.1 reichen am 14. April Beschwerde bei der Europäischen Kommission ein gegen die Vorschrift im novellierten niedersächsischen Mediengesetz ${ }^{14}$, Regionalfenster im Bundesland selbst produzieren zu müssen. Mit dieser Regelung soll verhindert werden, dass etwa der Privatsender Sat.1 die Produktion dieses Fensterprogramms nach Berlin verlagert, wie es Sat.1 in Erwägung gezogen hatte. Die Beschwerde bei der EU wende sich „vorbeugend“ auch gegen mögliche ähnliche Vorschriften in künftigen anderen Landesmediengesetzen.

Der Bundestag beschließt am 29. April einstimmig eine für Journalisten relevante Änderung des Strafgesetzbuches (StGB). ${ }^{15}$ Nach dem neu gefassten Paragrafen 201a StGB wird künftig mit einer Haftstrafe von bis zu einem Jahr oder einer Geldstrafe bedroht, wer unerlaubte Bildaufnahmen von einer Person macht, die sich „in einer Wohnung oder einem gegen Einblick besonders geschützten Raum befindet" und mit der Aufnahme sowie Weitergabe (Veröffentlichung) den „höchstpersönlichen Lebensbereich“ der Betroffenen verletzt. Auch wenn die Aufnahmen zwar „befugt hergestellt“, aber „wissentlich unbefugt“" weitergegeben werden, soll die neue Strafrechtsnorm gelten. Bislang galt das Aufnahme- und Weitergabeverbot nur für das vertraulich gesprochene Wort, und das mit der Ausnahme, dass nicht bestraft werde, wer bei der Weitergabe berechtigte Interessen geltend machen könne. Eingeschränkt wird mit dem neuen Paragrafen 201a StGB nicht nur die Tätigkeit von Prominentenfotografen („Paparazzi“), sondern potenziell auch die Arbeit investigativer (Fernseh-)Journalisten. Journalistenverbände hatten vorab vor einer Verschärfung des so genannten Bildnisschutzes gewarnt. Die Gesetzesänderung richtet sich nicht vorrangig gegen Journalisten, sondern

12 Das Auskunftsersuchen mit dem Fallzeichen CP 232/2002 zu TV-Produktionstöchtern ist dokumentiert in epd medien 32/2004 vom 28. April 2004, S. $25 \mathrm{ff}$.

13 Das Auskunftsersuchen mit dem Fallzeichen CP 43/2003 zu staatlichen Beihilfen ist dokumentiert in epd medien 32/2004 vom 28. April 2004, S. $19 \mathrm{ff}$.

14 In Kraft getreten am 1. Januar 2004, abgedruckt in Funkkorrespondenz Dokumentation 16/2004 vom 13. April 2004, S. 3 ff.

15 36. StrÄndG vom 30.7.2004 (BGBl I S. 2012 ). 
gegen Spanner im privaten Bereich. Sie ist auch eine Reaktion auf neue Techniken wie kleine Foto-Handys, die es leicht machen, unentdeckt Aufnahmen anderer zu machen.

Die vier deutschsprachigen Suchmaschinen Abacho, Apollo7, crossbot und Sharelook kündigen am 10. Mai an, ihre Arbeit künftig an einen Verhaltenskodex binden zu wollen, um so zu mehr Transparenz im Netz beizutragen. Diese Transparenz hatte u. a. kürzlich die Bertelsmann Stiftung gefordert, nachdem sie in ihrer Studie „Wegweiser im Netz“ zu dem Schluss gekommen war, dass Suchmaschinen keine objektiven Werkzeuge seien, um Informationen aus dem elektronischen Netz herauszufiltern.

Das Bundeskartellamt genehmigt am 12. Mai die 90-Prozent-Mehrheit der SPD-Medienholding DDVG am Druck- und Verlagshaus Frankfurt am Main (DUV), dem Verlag der „Frankfurter Rundschau“ (FR). Die Karl-Gerold-Stiftung, bisherige Alleineigentümerin der DUV und damit der FR, hatte der DDVG 90 Prozent der Anteile verkauft und damit auch auf eine Sperrminorität verzichtet. Die Übernahme der Zeitung durch die Medienholding der politischen Partei begegnete in der Öffentlichkeit eher politischen als wettbewerblichen Bedenken.

Am 24. Mai startet das digitale Antennenfernsehen DVB-T im Gebiet Bremen/Unterweser und in den beiden Regionen Hannover/Braunschweig sowie Köln/Bonn, im Oktober geht DVB-T auch im Rhein-Main-Gebiet und im November in den Regionen Düsseldorf/Ruhrgebiet und Hamburg/Kiel an den Start. Vorreiter mit Bereich DVB-T war im November 2002 Berlin gewesen. Im Jahr 2010 soll in Deutschland der Umstieg von der analogen auf die digitale Technik in allen Bereichen des Rundfunks endgültig vollzogen sein. Die privaten Programme verzichten aus Kostengründen auf eine parallele analoge und digitale terrestrischen Verbreitung ihrer TV-Programme und stellen die analoge Verbreitung ein. Die Förderung der neuen Technik durch deutsche Medienbehörden wird von der Europäischen Kommission wettbewerbsrechtlich untersucht.

Die vier Betreiber des Kulturprogramms 3sat halten am 24. Mai in ihrer „Lindauer Erklärung “ fest, auch in Zukunft ihr Engagement für 3sat konsequent fortzuführen. 15 der 16 Ministerpräsidenten halten hingegen im Rahmen der Unterzeichnung des 8. Rundfunkänderungsstaatsvertrags im Oktober in einer Protokollerklärung fest, ARD und ZDF sollten prüfen, ob und inwieweit das Gemeinschaftsprogramm auf deutscher Seite in die alleinige Zuständigkeit des ZDF überführt werden kann. Während das ZDF 3sat gern allein übernehmen würde, lehnt die ARD einen Ausstieg aus 3sat kategorisch ab. Das gemeinschaftlich von ZDF, ARD, dem ORF aus Österreich und der SRG aus der Schweiz gestaltete Drei-Länder-Programm feiert im Dezember 2004 sein 20-jähriges Bestehen.

Die niedersächsische SPD-Fraktion reicht am 25. Mai Klage beim Staatsgerichtshof in Bückeburg gegen die Novelle des niedersächsischen Mediengesetzes ein. Die Novelle, die am 10. Dezember 2003 mit den Stimmen der niedersächsischen Regierungsfraktionen CDU und FDP verabschiedet wurde und zum 1. Januar 2004 in Kraft trat, beschränkt eine mittelbare Beteiligung von Parteien an niedersächsischen Rundfunksendern auf unter 10 Prozent. Die SPD sieht ihre Partei mit dem neuen Mediengesetz bewusst wirtschaftlich geschädigt, da die SPD über ihre Medienholding Deutsche Druckund Verlagsgesellschaft mit 20,4 Prozent am hannoverschen Madsack-Verlag beteiligt ist, der wiederum Anteile an allen drei niedersächsischen Privatradios hält. Diese Beteilungen müssten bei Neu- oder Verlängerungslizenzierungen entsprechend reduziert werden.

Das Bundeskabinett beschließt am 26. Mai mit der 7. Novelle zum Gesetz gegen Wettbewerbsbeschränkungen (GWB) auch eine Neuregelung der Pressefusionskon- 
trolle. Bundeswirtschaftsminister Wolfgang Clement will sicherstellen, dass „auch unter den veränderten wirtschaftlichen Bedingungen und trotz der Konkurrenz durch neue Medien die Vielfalt der deutschen Presselandschaft erhalten bleibt“. Der Gesetzentwurf sieht eine moderate Anhebung der Schwellenwerte der Fusionskontrolle vor. Zusammenschlüsse von Verlagen sollen künftig erst ab einer Umsatzschwelle von zusammengerechnet 50 Mio. Euro (bisher 25 Mio.) genehmigungspflichtig sein. Das neue GWB erlaubt zudem Zeitungsverlagen unter bestimmten Bedingungen Zusammenschlüsse auch dann, wenn dadurch eine marktbeherrschende Stellung entstünde oder verstärkt würde. Die Lockerung der Pressefusionskontrolle bleibt jedoch heftig umstritten, sowohl das Bundeskartellamt als auch kleinere Verlage machen Bedenken geltend. Der zustimmungspflichtige Bundesrat fordert am 9. Juli zahlreiche Nachbesserungen am Gesetz. Die Verabschiedung zieht sich über das Jahresende hinaus. $^{16}$

Die Intendanten der Landesrundfunkanstalten einigen sich auf ihrer Sitzung am 7./8. Juni in Berlin auf den Entwurf einer Selbstverpflichtungserklärung zur Trennung von Werbung und Programm ${ }^{17}$. Sponsorhinweise sollen künftig nicht mehr Werbespots gleichen; mehr Zurückhaltung will sich die ARD auch beim Einsatz von TV-Gewinnspielen auferlegen.

In Rheinland-Pfalz beschließt das Kabinett am 8. Juni den Entwurf eines neuen Landesmediengesetzes, das das bisherige Landesrundfunkgesetz und das Landespressegesetz zusammenfasst und nach Verabschiedung im Landtag Anfang 2005 in Kraft treten soll. Geregelt wird darin u. a., dass nicht nur Hörfunk- und Fernsehsender, sondern auch Zeitungen in Zukunft ihre Beteiligungsverhältnisse in der jeweils ersten Ausgabe im Jahr im Impressum offen legen müssen. Die Zulassung für private Veranstalter darf in Zukunft nicht mehr automatisch um weitere zehn Jahre verlängert werden. Die Landeszentrale für private Rundfunkveranstalter (LPR) wird zudem in Landeszentrale für Medien und Kommunikation (LMK) umbenannt.

Der Hamburger Senat beschließt am 15. Juni eine Halbierung der Filmförderung auf 3,5 Mio. Euro. Der Beschluss trifft auf heftige Ablehnung in der Film- und Fernsehbranche. Studio Hamburg, das größte Produktionsunternehmen am Standort, droht am 17. Juni mit einem Teilwegzug nach Berlin. Auch die niedersächsische Landesregierung kündigt für 2005 Kürzungen der Finanzmittel an die Filmförderanstalt Nordmedia der Länder Niedersachsen und Bremen um knapp 1 Mio. Euro an. Für 2004 zahlte sie rund 2,8 Mio. Euro. Das Medienboard Berlin-Brandenburg hingegen gibt am 4. Oktober bekannt, dass die Stadt Berlin ihren Anteil an der Berlin-Brandenburg-Filmförderung für das Jahr 2004 um 3 Mio. Euro auf insgesamt 18,5 Mio. Euro aufstockt. Die Film- und Medienförderung der beiden Länder wurde erst 2004 in einem gemeinsamen Medienboard zusammengefasst. Die Gelder für die Filmförderungen betrugen zuvor 7,5 Mio. Euro pro Land.

Der rheinland-pfälzische Ministerpräsident Kurt Beck (SPD), auch Vorsitzender der Rundfunkkommission der Länder, regt am 16. Juni anlässlich eines Symposions zum 20jährigen Jubiläum des Privatfunks an, dass die Landesmedienanstalten auch Aufsichts-

16 Das Gesetz wird erst am 16. und 17. Juni 2005 von Bundestag und Bundesrat beschlossen und tritt rückwirkend zum 1. Juli 2005 in Kraft (BGBl I 2005, S. 1954). Ausgenommen von einer Anpassung bleiben jedoch die Regelungen zur Fusionskontrolle. Diese sollen zu einem späteren Zeitpunkt umgesetzt werden.

17 Das Sechs-Punkte-Papier der Intendanten ist dokumentiert in epd medien 47/2004 vom 19. Juni 2004, S. $19 \mathrm{ff}$. 
funktionen über ARD und ZDF übernehmen sollten. Peter Voß, Intendant des Südwestrundfunks, weist das Anliegen scharf zurück.

Die Deutsche Fußball-Liga GmbH und die Europäische Kommission einigen sich im April über ein neues Vermarktungsmodell für die Bundesliga. Ab der Saison 2006/2007 werden die Übertragungsrechte für die jeweils 306 Spiele der 1. und 2. Bundesliga in mehrere Pakete aufgeteilt und öffentlich ausgeschrieben. Die Rechte für Free-TV, PayTV, Internet und Mobilfunk können damit von mehreren Fernsehsendern und Medienunternehmen erworben werden. Ein ähnliches Modell gilt bei der Vermarktung der Champions League.

Das Bundeskartellamt untersagt Ende April den Einstieg der Betreiber der Heilbronner Lokalstation Radio Tom beim Reutlinger Privatsender Neckar-Alb-Radio. ${ }^{18}$ Die Ludwigshafener Medienunion würde über ihre Beteiligungsfirmen Moira und Südwestdeutsche Medienholding eine marktbeherrschende Stellung im Hörfunkmarkt der Region Stuttgart-Heilbronn erreichen.

Der Vorsitzende der Kommission für Jugendmedienschutz (KJM), Wolf-Dieter Ring, zieht am 22. Juni auf dem „Medienforum NRW“ Bilanz nach einem Jahr der Tätigkeit der KJM. Diese hat sich im ersten Jahr ihres Bestehens mit 360 Verstößen gegen straf- oder rundfunkrechtliche Bestimmungen im Internet befasst, 275 davon wurden abschließend bewertet. Zwei Drittel der geprüften Fälle hatten einen pornografischen Hintergrund, daneben spielte die Auseinandersetzung mit rechtsradikalen Inhalten in Internet-Angeboten eine herausragende Rolle. Die KJM ist seit dem Inkrafttreten des Jugendmedienschutz-Staatsvertrags am 1. April 2003 auch für das Internet die zentrale Aufsichtsstelle für den Jugendschutz und den Schutz der Menschenwürde.

Das Bundesverwaltungsgericht in Leipzig entscheidet am 23. Juni im Streit um die Stasi-Akten von Altbundeskanzler Helmut Kohl (CDU), dass Journalisten bei der Stasi-Unterlagenbehörde ohne Einwilligung des Betroffenen nur noch aus allgemein zugänglichen Quellen entstandene Akten einsehen dürfen. Forschern hingegen darf die Behörde weitere Akten herausgeben, sofern diese nicht den schutzwürdigen Interessen der Betroffenen entgegenstehen. Der Deutsche Journalisten-Verband (DJV) kritisiert, dass damit die Aufarbeitung der gesamtdeutschen Geschichte erschwert werde. Journalisten hätten künftig keine Möglichkeit, ohne Einwilligung der Betroffenen in Akten zu recherchieren.

Der Europäische Gerichtshof für Menschenrechte (EGMR) in Straßburg verurteilt Deutschland am 24. Juni wegen mangelhaften Schutzes der Privatsphäre in den Medien. ${ }^{19}$ Prinzessin Caroline von Hannover (geborene von Monaco) hatte wegen der unerlaubten Veröffentlichung von Fotos in mehreren deutschen Magazinen geklagt. Nach Auffassung des EGMR verstieß die bisherige deutsche Rechtspraxis gegen das Grundrecht auf Schutz des Familien- und Privatlebens, das in der Europäischen Menschenrechtskonvention verankert ist. Verleger- und Journalistenorganisationen kritisieren das Urteil heftig als „Rückschlag für die Pressefreiheit“ und fordern die Bundesregierung auf, Rechtsmittel dagegen einzulegen. Die Bundesregierung entscheidet jedoch am 1. September, keine Einwände gegen das „Caroline-Urteil“ des EGMR zu erheben. Das Urteil habe für die deutschen Gerichte keine bindende Wirkung, begründete Bundesjustizministerin Brigitte Zypries (SPD) die Entscheidung des Kabinetts, ausschlaggebend bleibe die Rechtsprechung des Bundesverfassungsgerichts. 
Der Bundesgerichtshof urteilt am 24. Juni, dass Werbeblocker für das Fernsehen rechtens sind. ${ }^{20}$ Der von der Koblenzer TC Unterhaltungselektronik AG (TCU) hergestellte Werbeblocker „Fernsehfee“ erschwere zwar die geschäftliche Tätigkeit des durch Werbung finanzierten Fernsehens, hier in Form des Klägers RTL, bedrohe es aber nicht existenziell. RTL müsse sich „den Herausforderungen des Marktes stellen“, es also letztlich hinnehmen, dass Wettbewerb und Innovation auch solche Geräte hervorbrächten. Mit seinem Urteil bestätigt der BGH eine Entscheidung des Berliner Kammergerichts aus dem Jahr 2001. Auf die zunächst erwogene Verfassungsbeschwerde gegen das BGHUrteil verzichtet RTL, kündigt aber an, gegen das modernisierte Nachfolgeprodukt der „Fernsehfee“ namens „Tivion 2“ auch weiterhin gerichtlich vorzugehen.

Die Europäische Kommission leitet am 14. Juli eine förmliche Prüfung im Zusammenhang mit den Umstellungskosten auf das digitale Antennenfernsehen (DVB-T) im Großraum Berlin-Brandenburg ein. ${ }^{21}$ Gegenstand des Verfahrens seien Ausgleichszahlungen, mit denen die Medienanstalt Berlin-Brandenburg (MABB) private Rundfunkveranstalter beim Umstieg auf DVB-T unterstützt habe. Die Kommission vermutet, dass es sich dabei um staatliche Beihilfen gehandelt habe, da die Telekom-Tochter T-Systems, die das DVB-T-Netz betreibe, durch diese Subventionen mittelbar begünstigt worden sei.

Der kommerzielle Hörfunksender Delta Radio aus Kiel verstößt nach Beschluss des für die Senderaufsicht zuständigen Medienrats der Unabhängigen Landesanstalt für neue Medien (ULR) vom 15. Juli mit seinem Gewinnspiel „Delta Radio sucht das geilste Liebespaar" gegen die geltenden Jugendschutzvorschriften. Die Kommission für Jugendmedienschutz (KJM) hatte das Spiel zuvor beanstandet, weil es insbesondere Kindern ein falsches Bild von Sexualität vermittle.

Die Europäische Kommission billigt die Fusion der Musiksparten von Bertelsmann und Sony zum weltweit zweitgrößten Musikproduzenten am 19. Juli ohne Auflagen. Die Behörde sei zu dem Schluss gekommen, dass es keine hinreichenden Gründe gegen den Zusammenschluss gebe, weitere Konzentrationstendenzen in der Musikbranche würden jedoch sorgfältig beobachtet und geprüft. Die Kommission habe die im Januar angemeldete Fusion sehr sorgfältig untersucht, da sich damit die Zahl der großen Musikproduzenten von fünf auf vier verringere, heißt es in einer Mitteilung der Kommission. Jedoch werde Sony BMG in Europa nicht die Nummer eins, diese Position werde weiter von Universal gehalten. Vor diesem Hintergrund sei geprüft worden, ob der Zusammenschluss eine marktbeherrschende Stellung für Sony BMG sowie die Unternehmen Universal, EMI und Warner Music schaffe oder verstärke. BMG gehört zu dem Medienkonzern Bertelsmann und umfasst die Labels Arista, Jive, Zomba und RCA. Sony Corp of America ist Teil des japanischen Elektronikkonzerns Sony. Zur Sony $\mathrm{Mu}-$ sic Entertainment gehören unter anderem Columbia, Epic und Sony Classical. Das neue Unternehmen Sony BMG, das je zu Hälfte von Bertelsmann und Sony gehalten wird, hat seinen Sitz ab 1. August in New York.

Am 19. Juli präsentiert die ARD eine Untersuchung zu den „rundfunkbezogenen Aktivitäten des Ministeriums für Staatssicherheit (MfS) der ehemaligen DDR in der DDR sowie in der Bundesrepublik Deutschland“22. Der Studie des Forschungsverbun-

20 BGH AZ I ZR 26/02. Das Urteil ist dokumentiert in epd medien 70/2004 vom 8. September 2004, S. $19 \mathrm{ff}$.

21 Das Schreiben der Kommission an die Bundesregierung vom 14. Juli 2004, mit dem das offiziell eingeleitete Verfahren begründet wird, ist dokumentiert in epd medien 68/2004 vom 1. September 2004, S. $12 \mathrm{ff}$. 
des SED-Staat der Freien Universität Berlin zufolge ist der Einfluss des MfS auf die ARD-Sender nur minimal gewesen.

Die niedersächsische Staatskanzlei und RTL geben am 2. August bekannt, dass sie sich im bereits über zehn Jahre andauernden Streit um ein Bußgeldverfahren außergerichtlich auf die Zahlung von 12 Mio. Euro (und damit 2 Mio. weniger als ursprünglich vorgesehen) durch den Sender geeinigt haben. RTL nimmt dafür seine Verfassungsbeschwerde zurück. Das Bußgeld war 1993 von der Landesmedienanstalt Niedersachsens verhängt worden, weil der Sender seine Fernsehreihe „Der große TV-Roman“ häufiger als erlaubt mit Werbeblocks unterbrochen hatte.

Der 6. Bericht zur Durchführung der EU-Fernsehrichtlinie, der im August vorgestellt wird, bilanziert für die Jahre 1999-2002 einen Zwei-Drittel-Anteil von europäischen Film- und TV-Produktionen im öffentlich-rechtlichen und privaten Fernsehen der 15 alten EU-Mitgliedsländer. Bei den 26 untersuchten deutschen Fernsehsendern lag der Anteil europäischer Produktionen mit 59,8 Prozent im Jahr 2002 klar unter dem EU-Durchschnitt (66,1 \%).

Die Kommission für Jugendmedienschutz (KJM) teilt am 9. August mit, dass die Privatsender MTV und RTL 2 mit der Ausstrahlung von Unterhaltungssendungen zu Schönheitsoperationen im Vor- und Abendprogramm gegen die Jugendschutzvorschriften verstoßen. Bei MTV wurden drei Folgen des US-Formats „I want a famous face“ beanstandet, bei RTL eine Folge von „Big Brother“, in der ein plastischer Chirurg Teilnehmerinnen der Show auf mögliche Schönheitsoperationen hin untersuchte. Durch die unkritische Darstellung von Schönheits-OPs könnten Jugendliche in der Bewältigung zentraler Entwicklungsaufgaben gefährdet sein, die Folgen hätten daher erst zu späterer Uhrzeit ausgestrahlt werden dürfen.

Das Bundeskartellamt untersagt Gruner+Jahr $(\mathrm{G}+\mathrm{J})$ am 9. August die Herausgabe der deutschsprachigen Ausgabe des Wissenschaftsmagazins „National Geographic“, die der Verlag bereits seit 1999 herausbringt. G+J hatte dafür 1998 mit dem spanischen Unternehmen RBA Publicaciones Internacionales in Barcelona ein paritätisches Gemeinschaftsunternehmen gegründet. Im Sommer 2003 stellte der Verlag beim Bundeskartellamt eine Voranfrage zur Übernahme der alleinigen Kontrolle über das Gemeinschaftsunternehmen. Nach Ansicht des Bundeskartellamts stellt der Lizenzerwerb jedoch einen Zusammenschluss dar, weil G+J das Kartellamt 1998 nicht über den beabsichtigten Lizenzerwerb informiert hatte. Nach Ansicht des Bundeskartellamts verfügte G+J bereits vor dem Zusammenschluss mit „Geo“ und „P.M“ auf dem Lesermarkt für populäre Wissenszeitschriften mit mehr als 60 Prozent über eine marktbeherrschende Stellung. Mit der deutschen Ausgabe von „National Geographic“ habe sich der Marktanteil auf rund 75 Prozent erhöht. G+J kündigt an, der Verlag werde Beschwerde gegen diese Entscheidung einlegen.

Der Vorsitzende der Kommission zur Ermittlung der Konzentration im Medienbereich (KEK), Peter Mailänder, wirft den Landesmedienanstalten am 18. August anlässlich der Vorstellung des 7. KEK-Jahresberichts ${ }^{23}$ vor, die Zusammenarbeit zu verweigern. Dadurch könne in einigen Fällen nicht festgestellt werden, ob eine vorherrschende Meinungsmacht vorliege. Die KEK reicht wegen mangelnder Auskunftsbereitschaft der BLM und der LfM Rechtsaufsichtsbeschwerde ein.

22 Eine Kurzfassung der Studien ist dokumentiert in Funkkorrespondenz 32/2004 vom 6. August 2004, S. $10 \mathrm{ff}$.

23 Der Jahresbericht mit u. a. einer ausführlichen Übersicht über die bundesweiten privaten TVAngebote und die Beteiligungsstrukturen ist im Internet unter www.kek-online.de verfügbar. 
Die Thüringer Landesmedienanstalt (TLM) lehnt eine Fusion mit den Medienanstalten in Sachsen und Sachsen-Anhalt ab. Eine Zusammenlegung würde den rundfunkrechtlichen, medienpolitischen und standortpolitischen Einfluss Thüringens schmälern, heißt es in einer am 1. September von der TLM-Versammlung veröffentlichten Erklärung. Die Ministerpräsidenten von Sachsen, Sachsen-Anhalt und Thüringen hatten im Rahmen einer „Initiative Mitteldeutschland“ eine Fusion der Landesmedienanstalten angeregt. Dazu sollte bis Ende September in den Staatskanzleien der Länder ein Prüfbericht erarbeitet werden.

Die Landesanstalt für Kommunikation Baden-Württemberg (LfK) und die Bayerische Landeszentrale für Medien (BLM) erarbeiten einen „Leitfaden für TV-Gewinnspiele“24, der am 9. September zustimmend von der Gemeinsamen Stelle Programm, Werbung und Medienkompetenz (GSPWM) zur Kenntnis genommen wird. Der Leitfaden soll die einheitliche, praktische Regulierung von TV-Gewinnspielen ermöglichen.

Die Bundesregierung billigt am 22. September einen Entwurf des Gesetzes zur akustischen Wohnraumüberwachung von Bundesjustizministerin Brigitte Zypries. Gegen einen ersten Gesetzentwurf hatten im Juli Journalisten- und Verleger-Verbände protestiert, woraufhin die Bundesjustizministerin Änderungen versprochen hatte. Die Verfassungsrichter hatten die seit 1998 gültige Praxis der akustischen Wohnraumüberwachung (auch „Großer Lauschangriff“ genannt) im März als verfassungswidrig eingestuft. Nach dem neuen Gesetzentwurf soll die akustische Wohnraumüberwachung nur noch angeordnet werden dürfen, wenn auf Grund „tatsächlicher Anhaltspunkte“ anzunehmen sei, dass keine Äußerungen aus der absolut geschützten Privatsphäre erfasst würden. Das Bundesjustizministerium geht daher davon aus, dass Gespräche in Privatwohnungen künftig „live mitgehört“ werden müssten und der Aufwand für die Strafverfolgungsbehörden deutlich größer werde. Abhörmaßnahmen sollen nur noch bei Straftaten mit mindestens fünfjährigen Höchststrafen angeordnet werden können.

Am 29. September fordert der Musiker Heinz-Rudolf Kunze auf der gemeinsamen Anhörung der Bundestags-Enquêtekommission „Kultur in Deutschland“ und des Bundestagsausschusses „Kultur und Medien“ die Schaffung einer gesetzlichen Quote für deutschsprachige Musik im Hörfunk. In einer Unterschriftenaktion hatte er etwa 600 unterstützende Unterschriften gesammelt. Bei den Privatsendern sind, Emnid-Studien zufolge, weniger als ein Prozent der Titel deutschsprachig, bei den ARD-Radios seien es im Durchschnitt 1,5 Prozent. Die rotgrüne Mehrheit des Bundestags stimmt am 17. Dezember für einen Antrag der Regierungskoalition, nach dem sich die Hörfunksender freiwillig verpflichten sollten, $\mathrm{zu}$ mindestens 35 Prozent in Deutschland produzierte Pop- und Rockmusik zu senden. Während der ARD-Vorsitzende Jobst Plog den Bundestag weder für zuständig noch den Vorstoß für sachgerecht hält, begrüßt der Bundesverband der Phonographischen Wirtschaft den Bundestagsbeschluss. Die Hörfunkveranstalter lehnen eine solche Quote jedoch ab.

Die Ministerpräsidenten der Länder beschließen auf ihrem Jahrestreffen vom 6.-8. Oktober, dass die Rundfunkgebühren ab dem 1. April 2005 nur um 88 Cent pro Monat auf dann 17,03 Euro erhöht werden sollen. In dem Betrag ist ein Aufschlag von 7 Cent dafür enthalten, dass die Gebühren erst zum 1. April und damit 3 Monate später als vorgesehen steigen. Die unabhängige Kommission zur Ermittlung des Finanzbedarfs der Rundfunkanstalten (KEF) hatte empfohlen, die Rundfunkgebühren um 1,09 Euro zu er-

24 Abgedruckt in Funkkorrespondenz 38/2004 vom 17. September 2004, S. 32. 
höhen und dies bereits zum 1. Januar. Angesichts der wirtschaftlichen Lage und der sozialen Einschnitte war dies mehreren Bundesländern zu viel, so dass die Länder erstmals von einem KEF-Vorschlag zur künftigen Gebührenhöhe abwichen. ARD und ZDF sollen nun ihren Personalbestand weiter reduzieren. Im ARD-Verbund sollen die neuen Landesrundfunkanstalten bis Ende 2008 von den insgesamt mehr als 21.600 Stellen 250 Stellen abbauen. Der Finanzausgleich zwischen den ARD-Anstalten bleibt bestehen, reduziert sich aber ab dem 1. Januar 2007 auf 1 Prozent des Nettogebührenaufkommens, von dem Radio Bremen künftig 54 Prozent und der Saarländische Rundfunk 46 Prozent erhalten.

Der Bundesgerichtshof (BGH) urteilt am 19. Oktober letztinstanzlich, dass Bildberichte aus dem Privatleben unter Umständen erlaubt sind. Im konkreten Fall ging es um eine Klägerin, die seit 2001 eine Beziehung zu dem früheren Ehemann der Schauspielerin Uschi Glas, Bernd Tewaag, unterhalte. Die Klägerin hatte erreichen wollen, dass den Zeitschriften „Das Neue“ (Heinrich Bauer Verlag), „Super Illu“ und „Freizeit-Revue“ (beide Burda) untersagt wird, weiterhin bestimmte Fotos aus ihrem Privatleben zu veröffentlichen, und hatte zunächst Recht bekommen. Weil Tewaag und die Klägerin sich zwischenzeitlich vor der Presse zu ihrer Beziehung bekannt und in diesem Zusammenhang auch hatten abbilden lassen, urteilt der BGH, dass die Klägerin, soweit sie ihre privaten Angelegenheiten selbst der Presse zugänglich gemacht hat, hinnehmen muss, dass hierüber berichtet wird. Die Veröffentlichung von privaten Bildern aus einem Zeitraum vor der Selbstdarstellung sei deswegen aber nicht gestattet.

Der deutsche Kabelverband fordert am 19. Oktober, die Kabelbelegungsvorschriften im deutschen Medienrecht zugunsten der großen Netzbetreiber zu ändern, damit diese selbst darüber entscheiden könnten, welche Programme sie über das analoge und digitale Kabel verbreiten wollen. Der Kabelverband vertritt seit Februar 2003 Kabelnetzbetreiber der Netzebene 3 (Kabel bis zum Hausverteilnetz), darunter Ish in NordrheinWestfalen, Kabel Baden-Württemberg, iesy in Hessen sowie Kabel Deutschland GmbH (KDG), die zusammen knapp 18 Mio. Kabelhaushalte erreichen.

Der Pay-TV-Programmanbieter Premiere reicht am 27. Oktober bei der Europäischen Kommission in Brüssel eine Beschwerde wegen Wettbewerbsverletzung ein. Er wirft dem öffentlich-rechtlichen Rundfunk die Bildung von Einkaufskartellen vor. Förmlich richtet sich die Beschwerde gegen die Europäische Rundfunkunion (EBU), die sich Mitte Juni für insgesamt 614 Mio. Euro die Rechte an den Olympischen Spielen bis 2012 sichern konnte, während Premiere leer ausging. Premiere reicht darüber hinaus eine Beihilfebeschwerde bei der EU ein und wirft ARD und ZDF vor, über die EBU mit Gebührenmitteln auch Sportübertragungsrechte für das Abonnementsfernsehen erworben zu haben, die sie gar nicht nutzen könnten. Beihilfen in Form von Rundfunkgebühren würden also genutzt, um private Wettbewerber zu behindern.

Der Deutsche Bundestag beschließt am 28. Oktober einstimmig das „Gesetz für die Rundfunkanstalt des Bundesrechts ,Deutsche Welle’ (DW)“. ${ }^{25}$ Es tritt zum 1. Januar 2005 in Kraft. Die Deutsche Welle soll danach weiter zur Vermittlung des Deutschlandbildes in Europa und der restlichen Welt beitragen sowie das Verständnis und den Austausch der Kulturen und Völker fördern, wobei das Internet explizit als dritte Programmsäule der Sendeanstalt verankert wird. Vor dem Hintergrund dieses offen formulierten Programmauftrags soll die DW jeweils für einen Zeitraum von vier Jahren ihre

25 Abgedruckt in Funkkorrespondenz Dokumentation 52/2004 vom 23. Dezember 2004, S. 3ff. 
Aufgabenplanung bestimmen und in eigener Verantwortung die Schwerpunkte der Arbeit setzen. Durch diese Selbstregulierung soll die journalistische Unabhängigkeit und die mittelfristige Planungssicherheit der Anstalt gestärkt werden.

Das Verwaltungsgericht München bestätigt am 4. November die Auffassung der Bayerischen Landeszentrale für neue Medien (BLM), dass der Musiksender MTV fünf vorliegende Folgen seiner sechsteiligen Sendereihe „Freak Show“ nur noch ab 23 Uhr erneut ausstrahlen darf. Die Folgen könnten für Kinder und Jugendliche entwicklungsbeeinträchtigend wirken. Die sechste Folge der bereits mehrfach ausgestrahlten Reihe dürfe überhaupt nicht mehr gesendet werden. MTV hatte die Reihe 2002 ins Programm genommen. Nachdem sich ein 14-Jähriger bei der Nachahmung eines Stunts aus der Freak-Show schwer verletzt hatte, war die zuständige BLM eingeschritten, um Wiederholungen zu verhindern.

Die Thüringer Landesmedienanstalt teilt am 11. November mit, dass sie die finanzielle Unterstützung für Digitalradio und digitales Antennenfernsehen vorzeitig einstellen werde. Die Rücklagen für die technische Förderung seien „weitgehend aufgebraucht". Der Digitalradiobetrieb werde nur noch bis Mitte und nicht mehr bis Ende 2005 unterstützt, für bundesweite Fernsehprogramme stellte die TLM-Versammlung im Rahmen des Haushalts 2005 kein Geld für den Betrieb digitalen Antennenfernsehens (DVB-T) zur Verfügung. Wenig später spricht sich auch der Medienrat der Medienanstalt Berlin-Brandenburg (MABB) in einem am 23. November veröffentlichten Positionspapier für einen Ausstieg aus der DAB-Technik (= Digital Audio Broadcasting) aus. ${ }^{26}$ Der MABB-Medienrat begründet seine Forderung mit fehlender Marktakzeptanz und technischen Mängeln. Das digitale Radio der Zukunft müsse auf anderen Kanälen seinen Weg zum Hörer finden. Die Direktorenkonferenz der Landesmedienanstalten (DLM) bekennt sich hingegen auf ihrer Sitzung am 14. Dezember zur Digitalisierung der Hörfunkübertragungswege und bekräftigt mehrheitlich „die bundesweite Position“ zu DAB. Um DAB weiter zu befördern, wollen sich die Landesmedienanstalten für eine gemeinsame Initiative mit den öffentlich- rechtlichen und privaten Anbietern sowie der Endgeräte-Industrie einsetzen. Auch die Medienkommission der Landesanstalt für Medien NRW (LfM) hält am 17. Dezember am DAB-Standard für die Digitalisierung des Hörfunks fest.

Am 15./16. November verabschieden die medienpolitischen Sprecher der SPDLandtagsfraktionen aus Baden-Württemberg, Bayern, Nordrhein-Westfalen und Rheinland-Pfalz in Stuttgart eine Erklärung ${ }^{27}$, in der sie vor dem Ausstieg aus DAB warnen. Sie nehmen gleichzeitig eine Erklärung aller für Medienfragen zuständigen Abgeordneten der SPD-Fraktionen des Bundes, des Abgeordnetenhauses der Bürgerschaften, der Landtage sowie im Europäischen Parlament zu den Perspektiven der europäischen Medienpolitik ${ }^{28}$ an.

Eine Novelle des „Gesetzes über den Westdeutschen Rundfunk Köln“ (WDR-Gesetz) wird am 25. November mit der Stimmenmehrheit der Regierungsfraktionen von SPD und Grünen verabschiedet. Sie sieht u. a. die Einrichtung einer „Publikumsstelle“ im Sender für Beschwerden und Anregungen von Hörern und Zuschauern vor sowie eine Stärkung des programmbegleitenden Online-Angebots des WDR. Der WDR selbst

26 Abgedruckt in Funkkorrespondenz Dokumentation 52/2004 vom 23. Dezember 2004, S. $23 \mathrm{ff}$. und in epd medien 23/2004 vom 27. November 2004, S. $21 \mathrm{ff}$.

27 Abgedruckt in Funkkorrespondenz Dokumentation 52/2004 vom 23. Dezember 2004, S. 20.

28 Abgedruckt in Funkkorrespondenz Dokumentation 52/2004 vom 23. Dezember 2004, S. $21 \mathrm{ff}$. und in epd medien 91/2004 vom 20. November 2004, S. $8 \mathrm{ff}$. 
lehnt das neue Beschwerdemanagement ab, weil es „weder notwendig noch zielführend“ sei.

Die SPD-Medienkommission fordert am 29. November eine Internet-Entwicklungsgarantie für ARD und ZDF. Die Kommission, die sich im Frühjahr neu konstituiert hatte und vom rheinland-pfälzischen Ministerpräsidenten Kurt Beck geleitet wird, ist der Auffassung, dass es den öffentlich-rechtlichen Rundfunkanstalten möglich sein müsse, mit ihren Internet-Angeboten „auf das veränderte Mediennutzungsverhalten angemessen zu reagieren und programmbegleitende Angebote anzubieten“. Entschieden weist die Kommission damit auch Überlegungen des niedersächsischen Ministerpräsidenten Christian Wulff (CDU) zurück, der sich zuvor dafür ausgesprochen hatte, Werbung und Sponsoring im öffentlich-rechtlichen Rundfunk schrittweise auf Null zu reduzieren.

Die Freiwillige Selbstkontrolle Multimedia-Diensteanbieter (FSM) wird am 1. Dezember unter Bedingungen und Auflagen als Einrichtung der Freiwilligen Selbstkontrolle von der Kommission für Jugendmedienschutz (KJM) anerkannt. Mitglieder der FSM sind u. a. Internetanbieter wie AOL Deutschland und T-Online. Besteht bei Angeboten eines der Mitglieder ein Verdacht auf einen Verstoß gegen Jugendschutzbestimmungen, muss sich nun zunächst die FSM damit befassen.

Der niedersächsische Ministerpräsident Christian Wulff (CDU) fordert am 3. Dezember, dass der Norddeutsche Rundfunk (NDR) seine Berichterstattung aus Niedersachsen ausweiten soll. Zugleich solle der aus 58 Mitgliedern bestehende NDR-Rundfunkrat verkleinert werden. Wulff schließt zur Umsetzung seiner Forderungen auch eine Kündigung des Staatsvertrags nicht aus. Der NDR-Staatsvertrag gilt noch bis 2007 und müsste spätestens am 28. Februar 2005 gekündigt werden, da er sich sonst automatisch um weitere fünf Jahre bis 2012 verlängert. NDR-Intendant Jobst Plog zeigt sich verhandlungsbereit, die „Brechstange der Staatsvertragskündigung“ dürfe aber nicht eingesetzt werden. ${ }^{29}$

Die Stuttgarter Verlagsgruppe Georg von Holtzbrinck legt im Dezember Beschwerde beim Bundesgerichtshof (BGH) ein, um die „Berliner Zeitung“ doch noch übernehmen zu können. Das Düsseldorfer Oberlandesgericht hatte Ende Oktober eine Beschwerde von Holtzbrinck gegen die Entscheidung des Bundeskartellamts zurückgewiesen und die Ansicht des Kartellamts bestätigt, dass der Holtzbrinck-Verlag, der in Berlin bereits den „Tagesspiegel“ besaß, durch den Erwerb der „Berliner Zeitung“ eine marktbeherrschende Stellung auf dem Berliner Abonnementzeitungsmarkt erreichen würde. Daran habe auch der Verkauf des „Tagesspiegel“ an den ehemaligen Holtzbrinck-Manager Pierre Gerckens nichts geändert. Der Kartellsenat folgte der Auffassung des Kartellamts, dass Gerckens „die an ihn übertragenen Geschäftsanteile nur treuhänderisch verwalten und das wirtschaftliche Risiko des Erwerbs bei Holtzbrinck verbleiben sollte“. Holtzbrinck hatte den Berliner Verlag im Juni 2002 von Gruner + Jahr $(\mathrm{G}+\mathrm{J})$ übernommen. Die Geschäfte der „Berliner Zeitung“ werden derweil weiter von $\mathrm{G}+\mathrm{J}$ geleitet.

Der Bundesgerichtshof (BGH) bestätigt am 16. Dezember das Strafurteil des Landgerichts München I gegen die EM.TV-Gründer Thomas und Florian Haffa und verwirft damit die von den beiden Ex-Vorständen betriebene Revision. ${ }^{30}$ Damit bleibt es bei den vom Münchner Landgericht im April 2003 verhängten Geldstrafen in Höhe von

29 Die Kündigung ist nicht erfolgt.

30 Az. 1 StR 420/03. 
1,2 Mio. Euro für Thomas Haffa und 240.000 Euro für seinen jüngeren Bruder Florian. Beiden war zur Last gelegt worden, die wirtschaftliche Lage ihres Konzerns im August 2000 bewusst positiver als tatsächlich gegeben dargestellt zu haben, um so den Kurs der Aktien nach oben zu treiben.

Nordrhein-Westfalens Ministerpräsident Peer Steinbrück fordert am 20. Dezember umfassende Reformen der öffentlich-rechtlichen Rundfunkanstalten in Deutschland und bietet sich als Vermittler in Gesprächen mit ARD, ZDF und der EU-Kommission an. Der Vorsitzende der Rundfunkkommission der Bundesländer, der rheinland-pfälzische Ministerpräsident Kurt Beck (SPD), kündigt daraufhin Rundfunkgespräche der Länder mit der EU-Kommission an.

\section{Medienunternehmen}

Mit Jahresbeginn startet der private Nachrichtensender N24 eine Kooperation mit dem internationalen Wirtschaftssender CNBC Europe. Die deutschsprachigen CNBC-Reporter berichten mehrfach täglich bei N24 über das Geschehen an der Frankfurter Börse.

RTL übernimmt zu Jahresbeginn die Mehrheit der Anteile (insg. 50,2 \%) beim Nachrichtensender n-tv mit der Übernahme der 1,6-Prozent-Anteile des Verlegers Norman Rentrop. Das Kartellamt und die KEF hatten der Übernahme zugestimmt.

Am 29. Februar startet der Rundfunk Berlin-Brandenburg (RBB) sein drittes Fernsehprogramm rbb fernsehen. Regional unterschiedliche Angebote gibt es nur noch für die Nachrichtensendungen „Berliner Abendschau“ (früher SFB 1) und „Brandenburg aktuell“ (ORB Fernsehen). Am 1. Mai 2003 waren der Ostdeutsche Rundfunk Brandenburg (ORB) und der Sender Freies Berlin (SFB) zum neuen Rundfunk Berlin-Brandenburg (RBB) fusioniert. Im Bereich des Hörfunks waren im Dezember aus den Programmen beider fusionierten Sender zwei Klassikwellen zusammengelegt worden.

Das Medienunternehmen EM-TV teilt am 27. März mit, dass es die 50 Prozent der insolventen Kirch Media am 1998 gemeinsam gegründeten Kinderprogrammvermarkter Junior TV übernommen hat. EM-TV selbst hatte Ende Februar eine Insolvenz des börsennotierten Unternehmens nur knapp vermeiden können.

Die Firma des ehemaligen Kirch-Managers Jan Mojto, EOS Distribution GmbH, erwirbt am 29. März die Rechtehandelsfima Beta Film GmbH inklusive der dazu gehörenden Filmbibliothek (mit ca. 5000 Filmen) der ehemaligen Kirch-Gruppe. 2003 hatte Moijto bereits die Musikproduktionsfirma Unitel aus der Kirch-Konkursmasse erworben.

Die Radioholding Regiocast erhöht im Frühjahr ihre Anteile am Privatradio Landeswelle Thüringen auf 26 Prozent (größter Gesellschafter bleibt die Ludwigshafener MOIRA Rundfunk GmbH mit $46 \%$ ). Gleichzeitig übernimmt die Verlagsgesellschaft Madsack über seine Beratungsfirma teleconsult beim Privatradio Antenne Thüringen die Anteile des „Thüringer Wochenblattes“ und wird mit 12 Prozent der Anteile drittstärkster Gesellschafter nach der AVE-Gruppe (RTL) und der Suhler Verlagsgesellschaft des Süddeutschen Verlags. Entsprechende Genehmigungen erteilt die Thüringer Landesmedienanstalt (TLM) am 31. März. Antenne Thüringen kommt auf durchschnittlich 213.000 Hörer pro Stunde, die Landeswelle auf 117.000 Nutzer. Die Verlagsgesellschaft Madsack gehört zu 20,4 Prozent der SPD-Medienholding Deutsche Druck- und Verlagsgesellschaft (DDVG).

In Hamburg geht am 2. April der Bürger- und Ausbildungskanal Tide mit einem 
Fernseh- und einem Radioprogramm auf Sendung. Tide ist der Nachfolger des am 30. Juni 2003 eingestellten Offenen Kanals der Hansestadt, der mit dem neuen Hamburgischen Mediengesetz abgeschafft wurde.

Die Münchner Produktionsfirma Focus TV einigt sich im April mit der Firma DCTP darauf, dass Focus TV ab Juli einmal im Monat auf einem Sendeplatz der DCTP auf Vox sendet, ab Juli 2005 soll das Programm zusätzlich einmal im Monat einen Sendeplatz bei Sat.1 erhalten. Focus TV hatte im vergangenen Jahr bei verschiedenen Verwaltungsgerichten gegen die Vergabe der Sendezeit für unabhängige Dritte durch die zuständigen Landesmedienanstalten geklagt, die Klagen aber später zugunsten von Verhandlungen mit DCTP, der Produktionsfirma von Alexander Kluge, zurückgezogen. Die DCTP veranstaltet seit Mai 1988 als unabhängiger Dritter Fensterprogramme von insgesamt zwei Stunden pro Woche bei RTL und insgesamt 1,25 Stunden pro Woche bei Sat.1.

Ab 3. Mai sendet der zuvor in München ansässige Musikfernsehsender MTV aus Berlin. In der Hauptstadt haben bereits die Musikkonzerne Sony und Universal ihren Deutschlandsitz. Im September findet zudem erstmals Europas größte Musikmesse, die Popkomm, in Berlin statt in Köln statt.

Guillaume de Posch, bislang Chief Operating Officer (COO) der ProSiebenSat.1 Media AG, wird zum 1. Mai neuer Vorstandsvorsitzender des Medienkonzerns und löst damit Urs Rohner ab 14/29.

Das Hamburger Verlagshaus Gruner+Jahr verkauft seine 14-täglich erscheinende Fernsehzeitschrift „TV Today“ zum 1. Mai an den Hamburger Unternehmer Hans-Georg Barlach. Barlach hatte bereits 1999 gemeinsam mit dem Unternehmer Frank Otto die Boulevardzeitung „Hamburger Morgenpost“ von G+J übernommen; inzwischen ist er Alleinbesitzer der Zeitung. Ende September werden 68 von 108 festen Mitarbeitern gekündigt. „TV Today“ hatte gegenüber dem Vorjahr mehr als 10 Prozent der Auflage verloren (763.427 Exemplare im 2. Quartal 2004 nach IVW).

Die Deutsche Telekom AG verkauft am 5. Mai an der Börse in Paris etwa die Hälfte ihrer Anteile am Satellitenbetreiber SES Global für rund 260 Mio. Euro. Dieses Geld soll nun dem weiteren Schuldenabbau dienen. Die Telekom hält ihre SES-Beteiligung über zwei Gesellschaften, die GSH Global Satelliten-Beteiligungs-Holding GmbH und die DT-Satelliten-Holding $\mathrm{GmbH}$, die nun zusammen noch über eine wirtschaftliche $\mathrm{Be}-$ teiligung von etwa 7 Prozent und gut 5 Prozent der Stimmrechte verfügen.

Nach monatelangen Verhandlungen werden Ende Mai 70 Prozent der Anteile am Ballungsraumfernsehsender Hamburg 1 (HH1) von der Almond Media Beteiligungs $\mathrm{GmbH}$, einer Firma von vier leitenden HH1-Mitarbeitern, übernommen. Die Deutsche Fernsehnachrichtenagentur (DFA), die den Sender aus der Konkursmasse der insolventen Kirch Media erworben hatte, scheidet damit als Gesellschafterin aus. Der AxelSpringer-Verlag (ASV) hatte ursprünglich sämtliche Anteile übernehmen wollen und dafür auch bereits die Zustimmung des Bundeskartellamts erhalten, nicht aber die medienrechtliche Zustimmung der Hamburgischen Anstalt für neue Medien (HAM). Der ASV erhöht seine Beteiligung nun lediglich von bislang 6,65 auf 30 Prozent.

Der ZDF-Fernsehrat berät am 8./9. Juli in Berlin u. a. das Thema der Kooperationen der Fernsehanstalt mit Partnern aus der Wirtschaft. Das ZDF will künftig weniger Kooperationen mit Dritten im Programmbereich eingehen. Als Vorlage für den Richtlinien- und Koordinierungsausschuss dient ein Zehn-Punkte-Katalog ${ }^{31}$ von Intendant Mar-

31 Dokumentiert in epd medien 53/2004 vom 10. Juli 2004, S. $23 \mathrm{ff}$. 
kus Schächter, der mit den geplanten Maßnahmen künftig „jeglichem Verdacht einer schleichenden Selbstkommerzialisierung wirkungsvoll entgegen treten will.

Die ARD-Intendanten vereinbaren am 7./8. Juni, die Sendeabwicklung für ihr Gemeinschaftsprogramm Das Erste ab 2005 zentral vom Hessischen Rundfunk in Frankfurt/Main aus zu steuern. Durch die Zentralisierung sollen pro Jahr knapp 2,3 Mio. Euro eingespart werden können. Die Standortwahl fiel auf den HR, weil dort die GlasfaserÜbertragungsleitungen des so genannten Hybnets zusammenlaufen, wodurch alle ARD-Sender über Breitbandtechnik miteinander verbunden sind.

Am 24. Juni gibt der US-amerikanische Medienkonzern Viacom bekannt, dass er die Mehrheit der Viva Media AG übernommen und den beteiligten 14 Großaktionären (u. a. die Time Warner-Tochter Turner Broadcasting System mit 30,6\% und Universal International Music mit 15,3 \%) 75,8 Prozent der Anteile abgekauft hat. Viacom erwirbt zudem auch die 49 Prozent der Gesellschafteranteile von VIVAplus von Time Warner. Die vier Musikfernsehprogramme MTV, MTV 2 Pop, VIVA und VIVAplus gehören damit zum selben Konzern. Zur Viva Media AG gehören darüber hinaus auch Musikkanäle in Polen, der Schweiz, Ungarn und den Niederlanden sowie das Produktionsunternehmen Brainpool GmbH (das u. a. die Shows von Anke Engelke und Stefan Raab herstellt). Das Bundeskartellamt stimmt der Übernahme am 6. August zu.

Die in Abwicklung befindliche NRW Medien GmbH stellt ihren Geschäftsbetrieb Ende Juni ein. Die Auflösung des Unternehmens, das u. a. für die Ausrichtung des Medienforums NRW zuständig war und Multimedia-Unternehmen in NRW ansiedeln sollte, war im September 2003 von der Landesregierung Nordrhein-Westfalen beschlossen worden, weil die NRW Medien GmbH zu teuer und ineffektiv gewesen sei.

Die Deutsche Welle (DW) unter Leitung von Intendant Erik Bettermann erarbeitet ein Konzept zur strategischen Neuausrichtung des Senders bis $2009^{32}$, das der Rundfunkrat am 2. Juli zustimmend zur Kenntnis nimmt. Für die gleiche Sitzung des Aufsichtsgremiums legt der Intendant ein Sparkonzept ${ }^{33}$ vor, das darlegt, wie der notwendige Personalabbau und Einschnitte im Programm umgesetzt werden können. Die Kürzungen des Bundeszuschusses für die DW von 269,5 Mio. Euro auf 261,4 Mio. (ab 2005) stellt den deutschen Auslandsrundfunk vor die Notwendigkeit, kurzfristig weitere Einsparungen in Millionenhöhe zu erzielen, zu denen u.a. ein baldiger Umstieg auf die digitale Kurzwelle (DRM) gehören soll.

Die Intendanten des Saarländischen Rundfunks (SR) und Südwestrundfunks (SWR), Fritz Raff und Peter Voß, unterzeichnen am 6. Juli eine Kooperationsvereinbarung, derzufolge die kostensparende Zusammenarbeit der beiden Sender in den Bereichen Hörfunk, Fernsehen (drittes Programm), in der Verwaltung und der Technik sowie im Orchesterbereich intensiviert werden soll. Eine Fusion sei nach Auffassung von Peter Voß zwar sinnvoll, aber auf absehbare Zeit politisch nicht durchsetzbar.

Die Landesanstalt für Kommunikation Baden-Württemberg (LfK) lehnt am 16. Juli den Antrag des privaten Fernsehsenders BTV4u auf Lizenzverlängerung ab. Das Vorläuferprogramm BTV hatte Ende Juli 2002 Insolvenz angemeldet und am 7. April 2003 eine einjährige Lizenz mit Auflagen erhalten. Lizenznehmer Tomas Hornauer war durch sein Telefondiensteunternehmen im Bereich der 0190-er Nummern und seine Nähe zu einer Sekte in die Kritik geraten.

32 Das Strategiepapier „Deutsche Welle 2009“ ist dokumentiert in Funkkorrespondenz 32/2004 vom 6. August 2004, S. 14ff. und in epd medien 55/2004 vom 17. Juli 2004, S. $21 \mathrm{ff.}$

33 Das Sparkonzept ist dokumentiert in Funkkorrespondenz 32/2004 vom 6. August 2004, S. $19 \mathrm{ff}$. und in epd medien 52/2004 vom 7. Juli 2004, S. $19 \mathrm{ff}$. 
Die Studio Babelsberg GmbH wird im Juli vom französischen Eigentümer Vivendi Universal an eine Investorengruppe um Carl Woebecken und Christoph Fisser verkauft. Das traditionsreiche, aber chronisch defizitäre Studio (Umsatz: rund 26 Mio. Euro) wird für den symbolischen Kaufpreis von 1 Euro verkauft; zusätzlich übernimmt Vivendi Altschulden in Höhe von rund 18 Mio. Euro. Der Finanzbedarf für die nächsten drei Jahre wird auf etwa 40 Mio. Euro veranschlagt.

Die Radio NRJ GmbH, die die deutschen Hörfunkbeteiligungen des französischen Medienkonzerns NRJ S.A. bündelt, gibt am 2. August die vollständige Übernahme des Stuttgarter Radio Energy nach Aufkauf der 80-prozentigen Beteiligung der Familie Weng bekannt. Die Landesanstalt für Kommunikation (LfK) stimmt der Übernahme zu.

Am 5. August vereinbaren die Nielsen Media Research International und die AGB Group die Bildung eines neuen gemeinsamen Unternehmens zur Fernsehreichweitenforschung unter dem Namen AGB Nielsen Media Research. Beide sind mit 50 Prozent an der neuen Firma beteiligt, die ab Jahresende Fernsehforschung für Kunden in 46 Staaten durchführt, die mehr als 70 Prozent der weltweiten Fernsehwirtschaft repräsentieren.

Giovanni di Lorenzo wechselt zum 15. August vom Berliner „Tagesspiegel“ in die Chefredaktion der Wochenzeitung „Die Zeit“. Michael Naumann und Josef Joffe, die bisher Chefredakteure und Herausgaber in Personalunion waren, fungieren künftig ausschließlich als Herausgeber. Sowohl der „Tagesspiegel“ als auch die „Zeit“ gehören zur Verlagsgruppe Georg von Holtzbrinck.

Die Hamburger Produktionsfirma Me, Myself \& Eye (MME) gibt am 16. August bekannt, dass sie rückwirkend zum 1. Januar 2004 die TV-Produktionsholding Moviement (München) übernommen hat. MME sei damit die größte unabhängige Produktionsgesellschaft im deutschen Fernsehmarkt. MME produziert hauptsächlich Musikfernsehsendungen für private und öffentlich-rechtliche Sender sowie das komplette Programm von MTV2 Pop. Die zu Moviement gehörenden Produktionsfirmen Filmpool (Köln) und Allmedia (München) produzieren u. a. Gerichtsshows und Krimis.

Der Musikkanal Onyx stellt seinen Sendebetrieb Ende August ein. Das Programm der französischen Gruppe AB geht ab 15. September als Natur- und Dokumentationskanal Terra Nova auf Sendung, der mit Dokumentationen und Magazinen „Interesse für Ökologie und Umweltfragen“ wecken möchte. Die zuständige Landesanstalt für Medien Nordrhein-Westfalen hatte der Programmschemaveränderung am 23. April zugestimmt.

Der zuvor in Berlin ansässige Nachrichtensender n-tv sendet ab dem 30. August aus dem Cologne Broadcasting Center, in dem bereits Vox und Super RTL untergebracht sind.

Der Spiegel-Verlag in Hamburg beteiligt sich zu einem Viertel am Berliner Hörbuchverlag „Der Audio Verlag (DAV)“. Spiegel-Verlagsleiter Matthias Schmolz erklärt am 30. August, der Einstieg in den „wachsenden Hörbuchmarkt“ sei für den SpiegelVerlag eine „strategische Investition, die unsere erfolgreichen Aktivitäten im Buchmarkt sinnvoll ergänzt“. Der DAV war 1999 ursprünglich vom Aufbau-Verlag gegründet worden, 2001 kamen der SWR und der WDR als neue Gesellschafter und Inhalte-Lieferanten hinzu. Bei der jetzt erfolgten Neuordnung im Gesellschafterkreis gab der bisherige Mehrheitsgesellschafter Aufbau 25 Prozent an den Spiegel-Verlag ab; der Buchverlag von Bernd F. Lunkewitz hält jetzt noch 26 Prozent, die SWR Holding und die WDR media audiogroup jeweils 24,5 Prozent.

Die NWZ Funk-Fernsehen GmbH, ein Tochterunternehmen des Verlages der Oldenburger „Nordwest-Zeitung“, übernimmt sämtliche Anteile am Hamburger Pri- 
vatradio 106!8 Rock 'n Pop (früher: Alsterradio) und damit auch das digitale Radioprogramm All Rock. Darüber hinaus hält die Nordwest-Medien Holding größere Anteile an den Sendern SAW in Sachsen-Anhalt (32,1 \%) und r.s.2 in Berlin (43\%) sowie 5,2 Prozent an Radio FFN in Niedersachsen. Der Vorstand der Hamburgischen Anstalt für neue Medien (HAM) genehmigt die Übernahme am 1. September.

Die ddp Nachrichtenagentur GmbH stellt am 7. September Antrag auf Eröffnung eines Insolvenzverfahrens. Am 8. November wird bekannt, dass die Nachrichtenagentur im Rahmen einer so genannten übertragenden Sanierung von der Starnberger Beteiligungsgesellschaft Arques Industries AG übernommen und fortgeführt und der Geschäftsbetrieb auf die neu gegründete ddp Deutscher Depeschendienst GmbH übertragen werden soll. Eine Voraussetzung der Übernahme ist ein vorübergehender rund 20prozentiger Gehaltsverzicht der ddp-Mitarbeiter. Lutz Schumacher bleibt Chefredakteur von ddp, gibt das Amt des Geschäftsführers jedoch an Arques-Vorstandsmitglied Martin Vorderwülbecke ab. Schumacher hatte ddp zusammen mit anderen als Geschäftsführer und Gesellschafter von der ProSiebenSat.1-Tochter SevenOne Intermedia übernommen. SevenOne hatte sich infolge der Kirch-Insolvenz von der erst 1999 übernommenen Nachrichtenagentur trennen müssen. Nach der Deutschen Presseagentur dpa gilt ddp als die zweitgrößte deutsche Nachrichtenagentur.

Der Pay-TV-Anbieter Premiere meldet am 4. Oktober erstmals über 3 Mio. Abonnenten und einen Nettogewinn von 15,4 Mio. Euro. Ende 2004 sind 3.247.172 Kunden unter Vertrag. Mit der Anmeldung einer Aktiengesellschaft beim Münchner Handelsregister im November bereitet sich der Sender auf den geplanten Börsengang im Jahr 2005 vor.

Marc Conrad wird zum 1. November neuer Geschäftsführer von RTL Television in Köln und Nachfolger von Gerhard Zeiler.

Die Bertelsmann AG teilt am 9. November mit, dass sie ihr operatives Ergebnis nach Ablauf von neun Monaten des Jahres 2004 mehr als hat verdoppeln können. Das Operating EBIT (Ergebnis vor Finanzergebnis, Steuern, Abschreibungen auf Firmenwerte sowie vor Sondereinflüssen) sei von 375 Mio. Euro im Vorjahreszeitraum auf 812 Mio. gestiegen. Der Umsatz habe sich im selben Zeitraum um 1,9 Prozent auf 12,0 Mrd. Euro erhöht (Vorjahr: 11,7 Mrd.). Außerordentliche Effekte aus dem seit 1. August 2004 bestehenden Gemeinschaftsunternehmen Sony BMG Music Entertainment seien im dritten Quartal noch nicht enthalten. Bertelsmann investierte in den ersten neun Monaten 588 Mio. Euro, zwei Mio. Euro weniger als im Vorjahr. Die Mitarbeiterzahl weltweit stieg bis Ende September auf 75.984 Mitarbeiter (Ende 2003: 73.221). Am gleichen Tag bilanziert auch der Medienkonzern Axel Springer ein um Sondereffekte bereinigtes Ergebnis vor Steuern, Zinsen und Firmenwertabschreibungen (EBITA) in den ersten neun Monaten des Jahres von 261,8 Mio. Euro. Die deutliche Zunahme gegenüber dem Vorjahresergebnis (163,7 Mio. Euro) begründet der Vorstandsvorsitzende Mathias Döpfner u. a. mit der Vergleichsvereinbarung, die Springer mit der insolventen Kirchmedia-Tochter Taurus TV geschlossen hatte. Der Konzern gab an, er habe den Umsatz um 2,4 Prozent im Vergleich zum Vorjahreszeitraum auf 1,83 Mrd. Euro gesteigert. Im internationalen Geschäft seien die Erlöse um 7,7 Prozent auf 267 Mio. Euro gestiegen. Axel Springer erwirtschafte inzwischen 14,6 Prozent seines Umsatzes im Ausland. Die Zahl der Mitarbeiter sank von 11.740 im Vorjahr auf 11.417.

Die ARD-Hauptversammlung - die Konferenz der Intendanten und Gremienvorsitzenden - beschließt am 30. November, dass der Bayerische Rundfunk (BR) ab 2005 die Geschäftsführung für die ARD übernehmen wird. BR-Intendant Thomas Gruber wird 
damit ab 1. Januar 2005 neuer ARD-Vorsitzender und Nachfolger des NDR-Intendanten Jobst Plog, der dieses Amt ab 1. Januar 2003 inne hatte.

Gruner+Jahr gibt am 30. November bekannt, dass der Konzern zum Jahresbeginn 2005 die Mehrheit an der Motor-Presse Stuttgart (MPS), dem Marktführer im Segment der Motorpublikationen, übernehmen wird. Die Mehrheitsbeteiligung ergibt sich durch die Übernahme der bisherigen Anteile der Würzburger Vogel Medien Gruppe (45,7 \%) an den Vereinigten Motor-Verlagen (VMV) in Stuttgart, die mit dem Motor-Presse-Verlag (MPV) gesellschaftsrechtlich zur künftigen Motor-Presse Stuttgart (MPS) zusammengeführt werden soll. G+ J wird an der künftigen Motor-Presse Stuttgart (MPS) mit 54,9 Prozent die Mehrheit halten. Die Motor-Presse Stuttgart werde unverändert als eigenständiges Unternehmen am Standort Stuttgart weitergeführt. In Deutschland ergänzten die Zeitschriften der Motor-Presse Stuttgart - wie „auto motor und sport“, „Motorrad“, „Men’s Health“ und „connect“ - das G+J-Angebot um das Motor-, Telekommunikations- und Männerzeitschriften-Segment. Darüber hinaus würden in zahlreichen Ländern die Marktchancen gestärkt.

Der ZDF-Fernsehrat genehmigt am 10. Dezember den Haushalt 2005, der einen Überschuss von 25,1 Mio. Euro vorsieht. Ein Fehlbetrag könne nur aufgrund zusätzlicher Einsparungen mit einem Gesamtvolumen von 31 Mio. Euro verhindert werden, sagt Intendant Markus Schächter. Der Haushaltsplan 2005 sieht im Betriebshaushalt Gesamterträge in Höhe von 1.874,6 Mio. Euro und Aufwendungen von 1.831,7 Mio. Euro vor. Die Aufwendungen reduzieren sich somit nach Darstellung des Senders gegenüber dem vergangenen Jahr um 79,5 Mio. Euro.

Der bundesweite private Klassiksender Klassik Radio geht am 15. Dezember an die Börse. Die Aktie, die mit einem Kurs von 6,05 Euro startet, kann sich bis 17. Dezember mittags in Frankfurt am Main auf 8,45 Euro steigern. Die Hamburgische Anstalt für neue Medien (HAM) hatte keine medienrechtlichen Bedenken gegen die Neuordnung der Eigentümerstruktur.

EM.TV verkauft am 15. Dezember eine seit 1999 bestehende 45-Prozent-Beteiligung an der Tele München Gruppe (TMG) an deren Hauptgesellschafter Herbert Kloiber. Die EM.TV AG hatte sich im Rahmen der im ersten Halbjahr 2004 abgeschlossenen Restrukturierung einer Wandelanleihe 2000/2005 der früheren EM.TV \& Merchandising AG zum Verkauf ihres TMG-Pakets verpflichtet. Werner E. Klatten, Vorstandsvorsitzender der EM.TV AG, erklärte, mit dem Verkauf der TMG-Anteile sei die letzte große Aufgabe der Sanierung von EM.TV erledigt.

Am 23. Dezember gibt Hubert Burda Media die Übernahme der Verlagsgruppe Milchstraße bekannt. Der Burda-Verlag, der seit Ende 1995 an Zeitschriften der Hamburger Verlagsgruppe beteiligt ist, übernimmt nun auch die Anteile der italienischen Mediengruppe Rizzoli Corriere de la Sera (RCS). Verleger Hubert Burda erklärt, die Zeitschriften der Milchstraße ergänzten das Portfolio von Burda „optimal“. Dirk Manthey, der Gründer der Verlagsgruppe Milchstraße, spricht im „Spiegel“ hingegen von einer „feindlichen Übernahme“ durch Burda. Nach Angaben von Rizzoli zahlte der Münchner Verlag 28 Mio. Euro an die italienische Verlagsgruppe. In der Verlagsgruppe Milchstraße erscheinen unter anderem die Zeitschriften „Fit for fun“, „Max“, „Amica“, „TV Spielfilm“ und „Tomorrow“.

Der Süddeutsche Verlag (SV) gibt am 23. Dezember bekannt, dass er sich wieder an der in Hof erscheinenden „Frankenpost“ beteiligen und in einem ersten Schritt 35 Prozent der Anteile von der SPD-Medienholding Deutsche Druck- und Verlagsgesellschaft (DDVG) zurückkaufen werde. Die DDVG war seit Anfang 2003 Alleineigentümerin der „Frankenpost“, nachdem der SV seine 70-Prozent-Beteiligung an der Zeitung hatte 
aufgeben müssen, weil sich die Südwestdeutsche Medienholding als neuer Gesellschafter am SV beteiligt hatte. Dies führte zu einer kartellrechtlich relevanten Überschneidung von Verbreitungsgebieten in der Region Hof/Plauen.

\section{Medienmärkte}

\subsection{Medienangebote / Medieninhalte Presse}

Im sächsischen Görlitz geht am 3. Mai die neue lokale Tageszeitung Görlitzer Allgemeine (GA) auf den Markt. Die Zeitung erscheint montags bis sonnabends im TabloidFormat und kostet 60 Cent. Nur sechs Wochen später muss sie ihr Erscheinen nach dem Rückzug von Geldgebern wieder einstellen.

Die Zeitungsverlage umwerben mit Neugründungen kleinformatiger Zeitungen im Tabloid-Format die Gruppe junger Nichtleser: Im Mai bringt die Lausitzer Rundschau, die zum Verlagshaus Holtzbrinck gehört, das Tabloid-Format 20 Cent heraus, das sich vorwiegend an eine ,junge, konsumorientierte Zielgruppe“ richte, die bisher wenig Zeitung lese. Ebenfalls im Mai bringt der Axel Springer Verlag in Berlin Welt Kompakt an die Kioske, ebenfalls im handlichen Kleinformat und für die junge Zielgruppe, für den Preis von 50 Cent. Im September wird Welt Kompakt im Ruhrgebiet und in München eingeführt, Köln folgt am 4. Oktober. Die Verlagsgruppe Handelsblatt (VHB), die zum Holtzbrinck-Konzern gehört, geht am 14. September mit der Tageszeitung News an den Start. Die ebenfalls im Tabloid-Format erscheinende Zeitung will Menschen zwischen 20 und 39 ansprechen und ist im Internet ab sechs Uhr morgens als elektronische Ausgabe herunterzuladen bzw. zunächst nur in Frankfurt Montag bis Freitag zu einem Preis von 50 Cent zu erwerben. Das Kölner Medienhaus M. DuMont Schauberg schließlich startet am 1. Oktober eine zusätzliche Zeitung im Kleinformat, den Kölner Stadt-Anzeiger Direkt, der sich an eine „Kernleserschaft im Alter von 20 bis 39“ richtet. Die Zeitung, die zum Preis von 50 Cent verkauft wird, erscheint von Montag bis Freitag. Außer den als erfolgreich geltenden Neugründungen der Berliner tageszeitung (taz) 1978 und der Financial Times Deutschland 2000 hatte es Jahrzehnte lang kaum Veränderungen auf dem deutschen Zeitungsmarkt gegeben.

Das Premiere-Programmheft Premiere Magazin wird mit der Juni-Ausgabe eingestellt; die andere Programmzeitschrift von Premiere, TV Kofler, wird bereits im April durch das in Kooperation mit dem Springer-Verlag herausgegebene TV-Digital ersetzt.

Mit der im Juni erscheinenden Nummer stellt der Axel Springer-Verlag die monatliche Frauenzeitschrift Allegra, die sich an jüngere Frauen richtet, ein. Angesichts des anhaltend schwierigen Anzeigenmarktes gebe es „keine ausreichende wirtschaftliche Perspektive für die Zeitschrift".

Ab August bietet die Frauenzeitschrift Brigitte eine E-Paper-Ausgabe an. Alle zwei Wochen erscheint das Heft mittwochs morgens noch vor Auslieferung der gedruckten Ausgabe im Netz. Die Online-Ausgabe, die sich vor allem an Menschen im Ausland richtet, ist für alle Brigitte-Abonnenten kostenlos. Wer nur das Online-Angebot abonniert, zahlt pro Stück 2,20 Euro - genauso viel wie für die Print-Ausgabe. Ab dem 23. Oktober ist auch Der Spiegel als E-Paper-Ausgabe erhältlich. Die aktuelle Ausgabe des digitalen Spiegel wird jeweils in der Nacht von Samstag auf Sonntag ins Internet gestellt und ist mit der Print-Ausgabe identisch, inklusive Fotos, Grafiken und Anzeigen. Die E-Paper-Ausgabe kostet als Einzelausgabe drei Euro, ebenso viel wie das gedruckte Heft, der Abonnementspreis beträgt 2,80 Euro. Nach Angaben des Bundesverbandes Deutscher Zeitungsverleger (BDZV) bieten Ende Oktober 41 Zeitungen in Deutschland E-Papers 
an. Die Einzelauflagen der E-Papers sind jedoch noch gering. Führend ist die Rhein-Zeitung, die 2001 zur Pionierin dieser neuen elektronischen Erscheinungsform wurde und inzwischen via Internet 2477 Exemplare verkauft. Zweitplatzierte in der Auflagenstatistik sind die Stuttgarter Zeitung/Nachrichten mit 675 Exemplaren.

Der Axel Springer Verlag und der Spiegel Verlag teilen am 6. August gemeinsam mit, dass sie zur klassischen deutschen Rechtschreibung zurückkehren. Alle zu den Verlagen gehörenden Publikationen - auch die Onlineangebote - stellen ihre Schreibweise um. Die beiden Verlage appellieren an andere Verlage und an die Nachrichtenagenturen, sich ihrem Schritt anzuschließen und dem Beispiel der Frankfurter Allgemeinen Zeitung (FAZ) zu folgen, die bereits am 1. August 2000 zur alten Rechtschreibung zurückgekehrt war. Ein Jahr zuvor, am 1. August 1999, hatten alle Agenturen und Zeitungen auf die neue Rechtschreibung umgestellt.

Die Zeit geht Mitte Dezember mit einem neuen, eigenständigen Wissensmagazin Zeit Wissen auf den Markt. In einer Auflage von 250.000 Exemplaren soll das Magazin zunächst vierteljährlich erscheinen. Auch die Süddeutsche Zeitung (SZ) erscheint am 11. Dezember erstmals mit einem eigenständigen Wissensmagazin, das im Zeitschriftenhandel, an Bahnhöfen und Flughäfen für vier Euro verkauft wird. Focus hatte Ende Oktober angekündigt, dass das neue Bildungsmagazin Focus Schule ab Januar 2005 sechsmal jährlich erscheinen solle.

Der Axel Springer Verlag und Hubert Burda Media geben am 9. Dezember bekannt, dass das Journal für die Frau im Januar 2005 an Burda verkauft und dort mit der Frauenzeitschrift freundin fusioniert werden soll. Während für Springer „die fehlende wirtschaftliche Perspektive des Titels in den vorhandenen Strukturen“ der Grund für den Verkauf darstellt, will der Burda Verlag mit der Fusion beider Zeitschriften „einen ähnlichen Typus Frau ansprechen“. Der Kauf steht noch unter dem Vorbehalt der Zustimmung des Kartellamts. Burda hatte im April mitgeteilt, dass der Gesamtumsatz 2003 erstmals über die Zwei-Milliarden-Grenze gesteigert werden konnte. Die Zeitschriften des Verlags trugen etwas mehr als eine Milliarde bzw. 70,7 Prozent zum Außenumsatz bei. Burda verlegt 253 Titel im In- und Ausland (2002 waren es 235) und hält auch 30 direkte und indirekte Beteiligungen an Radiosendern sowie an Focus TV.

\subsection{Medienangebote / Medieninhalte Rundfunk}

\subsubsection{Fernseben}

Der US-Nachrichtensender CNN stellt mit Jahresbeginn seine deutschsprachige Sendung „CNN Deutschland“ ein. Das 15-minütige Fenster war 1997 eingerichtet worden und werktags dreimal täglich zu sehen.

Die ab dem 9. Januar bei RTL ausgestrahlte Dschungelshow „Ich bin ein Star - Holt mich hier raus!“ erzielt Marktanteile über 40 Prozent bei den 14- bis 49-Jährigen und sorgt für viel Aufregung. Die für RTL zuständige Niedersächsische Landesmedienanstalt (NLM) sieht aber keinen Anlass für medienrechtliche Konsequenzen, ebenso wenig wie die Kommission für Jugendmedienschutz (KJM), die das Thema auf ihrer Sitzung am 21. Januar behandelt. Die Sendungen bewegten sich jedoch „nahe an der Grenze zum Verstoß“. Die öffentliche Aufregung wird u. a. dadurch forciert, dass die RTLShow von der Boulevardpresse, allen voran Bild, ausgiebig kommentiert wird.

Mit dem am 18. Januar in fünf bayerischen und drei tschechischen lokalen Fernsehprogrammen gestarteten europäischen Magazin „Perspektive Europa - Wir überschreiten Grenzen“ erhalten Zuschauer im bayerisch-tschechischen Grenzgebiet regelmäßig 
Informationen aus der Region. Das auf 25 Folgen à 30 Minuten konzipierte 14-tägliche Privatprojekt rechnet mit Produktionskosten von 605.000 Euro, die zur Hälfte von der EU und zu weiteren 15 Prozent von der Bayerischen Landeszentrale für neue Medien (BLM) getragen werden. Ein vergleichbares grenzüberschreitendes Fernsehprojekt gibt es seit Ende 2000 bereits beim öffentlich-rechtlichen Sender Phoenix und dem polnischen öffentlichen Fernsehsender TVP2 mit der Sendereihe „Beiderseits der Oder“.

RTL startet am 2. März die fünfte Staffel von „Big Brother“, bei der die Kandidaten gleich ein ganzes Jahr beobachtet werden sollen. Dem Gewinner winkt ein Preisgeld von 1 Mio. Euro.

Der deutschsprachige Tier- und Naturdokumentationskanal Animal Planet nimmt am 31. März seinen Sendebetrieb auf. Das 24-Stunden-Programm, ein Joint Venture zwischen dem US-amerikanischen Discovery Channel und dem britischen Sender BBC Worldwide, wird über die Premiere-Plattform vermarktet und ist zusammen mit einem Premiere-Paket für zusätzlich 3 Euro pro Monat zu beziehen.

Zum 1. April erhöhen sich die Abonnementsgebühren des Pay-TV-Senders Premiere um teilweise über 25 Prozent. Während die so genannten Bestandskunden bis Ende des Jahres nur 3 Euro monatlich mehr zahlen müssen, ist das Abo für Neukunden 5 Euro teurer. Ausgenommen von der Preiserhöhung ist das Gesamtpaket „Premiere komplett", das rund 250.000 Kunden abonniert haben.

Die Kabel Deutschland GmbH (KDG) startet ab April ein kostenpflichtiges digitales Programmbouquet in ihren Kabelnetzen. In dem Paket „Kabel Digital Basic“ können für 7 Euro monatlich bis zu 47 digitale Radiokanäle und zahlreiche digitiale Fernsehspartenprogramme, darunter auch das Einstiegsangebot „Premiere Start“ des PayTV-Senders Premiere, abonniert werden. Eine Vermarktungskooperation zwischen Premiere und KDG war am 13. Februar offiziell mitgeteilt worden. Am 27. September stellt die KDG ihr neues digitales TV-Programmpaket „Kabel Digital Home“ vor. Es beinhaltet 30 Pay-TV- und 47 Radioprogramme. Die monatliche Abo-Gebühr beträgt bei einjähriger Laufzeit 9 Euro.

Am 3. Mai startet bei TV NRW ein Regionalfenster für das Westmünsterland. Werktags wird zwischen 17 und 22 Uhr fünfmal ein rund zehnminütiges Programmfenster mit Nachrichten aus der Region zwischen den Städten Borken, Bocholt und Gronau ausgestrahlt. Ein weiteres Programmfenster in TV NRW gibt es seit Sommer 2003 bereits für den Raum Köln/Bonn.

Als Spartenkanal für Spielfilme geht am 13. Mai der digitale Sender Kinowelt TV auf Sendung. Das Pay-TV-Programm ist in den Premium-Paketen von visAvision (über Eutelsat), bei Kabel Deutschland („Kabel Digital basic plus“) und Primacom („Kabel!Vision“) zu empfangen. Kinowelt Television hatte bei der Hessischen Landesanstalt für Privaten Rundfunk (LPR) die Zulassung für die bundesweiten Fernsehspartenprogramme Kinowelt TV und Kinowelt TV Premium beantragt.

Am 17. Mai geht als Nachfolgeprogramm der im Dezember 2003 eingestellten „Harald Schmidt-Show" von Sat.1 die „Anke Late Night“ mit Anke Engelke auf Sendung. Nach schlechten Quoten und sinkenden Werbepreisen setzt Sat.1 die Late Night Show am 21. Oktober wieder ab. Harald Schmidt geht mit einer neuen Show am 23. Dezember in der ARD auf Sendung.

Das Fernsehjahr ist von zwei sportlichen Großereignissen - der Fußball-Europameisterschaft in Portugal und den Olympischen Sommerspielen in Athen - geprägt. Sowohl die Fußballübertragungen als auch die Olympischen Spiele werden weitgehend von den öffentlich-rechtlichen Sendern abgedeckt und bescheren ARD und ZDF sehr gute Quoten. Das Erste erzielt einen durchschnittlichen Marktanteil von 18,2 Prozent, 
das ZDF kommt auf 18,1 Prozent, wobei die Eröffnungsfeier (ZDF: 12,92 Mio. Zuschauer) und die Schlussfeier (ARD: 9,73) die höchsten Sehbeteiligungswerte erzielen.

Der Kochkanal TV Gusto startet sein Programm am 21. September. Der neue Spartensender wird in einem Bündel von insgesamt 30 digitalen Fernsehkanälen über das $\mathrm{Ka}$ belnetz der KDG sowie über einen digitalen Astra-Satelliten verbreitet.

Ab dem 3. Oktober geht das Dritte Fernsehprogramm des Hessischen Rundfunks (HR) mit neuem Logo unter dem Namen HR-Fernsehen auf Sendung.

Der amerikanische Geschichtsfernsehsender The History Channel startet am 15. November mit einer deutschen Programmversion, die über die Kabelplattform der KDG in 13 Bundesländern im Rahmen des neuen Programmpakets „Kabel Digital Home“ verbreitet wird. Ein maßgeblicher deutscher Programmteil wird vom ZDF bzw. dessen Vermarktungstochter ZDF Enterprises geliefert. Im Rahmen des digitalen privaten Spartenprogramms werden auf Basis einer Lizenzvereinbarung ZDF-Sendungen wie „Terra X“, „Sphinx“, „Imperium: Aufstieg und Fall großer Reiche“ und „Die Große Freiheit“ zu sehen sein. Das ZDF und der History Channel arbeiten bereits seit $1995 \mathrm{zu}-$ sammen, seit drei Jahren läuft sonntags das koproduzierte Magazin „History“ mit Guido Knopp.

Am 20. Dezember geht im Ballungsraum Stuttgart der Fernsehsender rtv auf Sendung. Das Programm sendet tagesaktuell im 30min.-Repeat-Format und ist im Kabel von etwa 550.000 Haushalten zu empfangen.

Über die Tsunami-Katastrophe in Südasien am 26. Dezember wird ausgiebigst berichtet. Eine Vielzahl von Spendenaufrufen der Fernsehsender, aber auch Verlage und Radiosender erbringen Rekord-Spendensummen.

\subsubsection{Radio}

Die Jugendwelle $H R X X L$ des Hessischen Rundfunks (HR) startet zu Jahresbeginn als populär ausgerichtetes Musikprogramm mit dem neuen Namen You FM. Am 30. August schließt der HR seine Hörfunkreform mit dem Relaunch seiner drei neu positionierten Radioprogramme HR1, HR4 und HR Info ab. Um die Hörerakzeptanz zu steigern, setzt der HR auf durchhörbare Programme, die in Abgrenzung und Ergänzung zueinander stehen sollen. Im September 2003 war bereits die Kulturwelle HR2 neu gestartet.

Seit Anfang des Jahres trägt die Radiowelle $M D R$ Kultur den neuen Namen $M D R F i$ garo, der symbolisieren soll, dass es sich bei dem Kulturprogramm um „lebendiges Radio" handle.

Das gemeinsam von Radio Bremen und NDR betriebene Nordwestradio wird ab Februar wieder analog über den Satelliten Astra 1 E ausgestrahlt. Seit Jahresbeginn war das Programm nur noch digital zu empfangen gewesen. Gemeinsam mit der Hamburger Zeitschrift mare starten Radio Bremen und der NDR am 1. Februar die neue monatliche Hörfunksendung „mare Radio“, die jeweils am ersten Sonntag im Monat von 11.05 Uhr bis 13 Uhr im Nordwestradio gesendet wird. Ähnlich wie die Zeitschrift widmet sich auch die Radiosendung dem Schwerpunkt Meer. Seit Januar 2001 produziert der NDR bereits die Fernseh-Sendung „mareTV“, die jeweils am ersten Donnerstag im Monat zur Hauptsendezeit um 20.15 Uhr zu sehen ist.

Am 2. April geht in Berlin das erste Radio zum Thema Auto auf Sendung: Das 24Stunden-Programm Roadradio des Unternehmens Media Four Media wird zunächst nur via Internet ausgestrahlt, der Wortanteil soll bei 60 Prozent liegen.

In Hamburg starten am 11. Mai zwei neue private digitale Hörfunkprogramme: $R a-$ 
dio Digital 1 (veranstaltet vom Hamburger Marktführer Radio Hamburg, wendet sich mit einem angestrebten Anteil von 60 \% deutschsprachiger Musik an die 14- bis 29Jährigen) und All Rock (veranstaltet vom Hamburger Alsterradio, mit Rock- und Popmusik der letzten 40 Jahre). Als Zuschuss zu den Verbreitungskosten erhalten die beiden neuen Sender von der HAM 80.000 Euro über drei Jahre. Ab dem 15. Juni wendet sich das Alsterradio unter dem neuen Namen 106!8 Rock 'n Pop an männliche Hörer zwischen 30 und 54 Jahren.

Ab dem 12. Juli heißt der Privatsender Hitradio Antenne Sachsen in Dresden Hitradio RTL - wir sind Sachsen. Er wendet sich mit einem Musikprogramm an die 25- bis 49-Jährigen. Im Februar hatte die Hörfunktochter der RTL Group die Mehrheit an dem Sender erworben.

Das ehemalige Frankfurter Business Radio (FBR) startet am 6. September unter dem neuen Namen Main FM. Ende 2003 war der französische Medienkonzern Lagardère über sein Tochterunternehmen Europa1 mit 40 Prozent bei FBR eingestiegen und hatte eine Konzeptänderung verlangt, bei der Wirtschaftsnachrichten nicht mehr im Vordergrund stehen.

Der Medienrat der Medienanstalt Berlin-Brandenburg vergibt am 8. Oktober eine UKW-Frequenz für Berlin und weitere Frequenzen für 15 Standorte in Brandenburg. Die bisher von Radio Multikulti (RBB) genutzte Frequenz 106,8 MHz, mit der rund 3,5 Mio. Hörer in Berlin und Potsdam erreicht werden können, teilen sich das private Kinderprogramm Radio Teddy (6-21 Uhr) und das Musikradio Motor FM (21-6 Uhr). Radio Teddy wird von den Journalisten Uwe Schneider und Ulrich Kühler sowie dem Miteigentümer des Filmpark Babelsberg, Friedhelm Schatz, veranstaltet. Hinter Motor FM steht der ehemalige Chef der Plattenfirma Universal Music, Tim Renner, der sein Programm zu einem Viertel mit deutschen Produktionen füllen will.

Am 6. Dezember startet der Sender Wilantis - das Wissensradio. Das über DAB, Internet und Astra verbreitete Hörfunkprogramm kann im Raum Stuttgart auch über UKW technisch von rund 500.000 Hörern empfangen werden und will „Wissen in verständlicher Form vermitteln“.

Das DAB-Hörfunkprogramm Klassik Digital Nord, ein 24-stündiges Gemeinschaftsprogramm der drei niedersächsischen Privatradios Hitradio Antenne Niedersachsen, Radio FFN und Radio 21 in Kooperation mit dem bundesweiten Musiksender Klassik Radio, wird zum Jahresende eingestellt, nachdem die Niedersächsische Landesmedienanstalt (NLM) im Oktober beschlossen hatte, die finanzielle Förderung in Höhe von monatlich knapp 25.000 Euro, die das Gros der technischen Verbreitungskosten trug, zu beenden. Auch im fünften Jahr des Regelbetriebs habe der Verbraucher die DAB-Empfänger nur zögerlich nachgefragt.

\subsection{Medienangebote / Medieninhalte Online}

Am 18. März startet das Musikvertriebssystem PhonoLine in Hannover anlässlich der Computermesse CeBIT. Die Plattform, die zum Auftakt rund 250.000 Musiktitel zum Runterladen anbietet, wird von der Telekom-Festnetzsäule T-Com als Dienstleister für die angeschlossenen Musikkonzerne betrieben und soll offen sein für alle Musikfirmen, die ihr Angebot integrieren wollen, und für alle Online-Händler, die diese Plattform als Basis für Musikangebote auf ihren eigenen Webseiten nutzen wollen. PhonoLine soll den Gratis-Tauschbörsen im Internet eine kommerzielle Alternative entgegensetzen. Bereits am 24. September wird die Plattform jedoch mangels Interesse wieder eingestellt. Ein früherer Versuch des Bertelsmann-Konzerns, mit Napster eine 
kostenlose Tauschbörse in einer Allianz zu kontrollieren und in eine kostenpflichtige Form zu überführen, war gescheitert, weil die Konkurrenten EMI und Vivendi eine 17-Milliarden-Dollar-Sammelklage wegen angeblicher Urheberrechtsverletzungen angestrengt hatten.

Am 23. April startet die Landesmedienanstalt Saarland (LMS) ein Internet-Portal programmbeschwerde.de, über das sich Hörer und Zuschauer online über private Radio- und Fernsehprogramme beschweren können. Nach einem Beschluss der Direktorenkonferenz wird das Portal im Herbst zu einer gemeinsamen Einrichtung aller Medienanstalten.

Der Bundesverband deutscher Zeitungsverleger verzeichnet im Juli 2004 insgesamt 633 Online-Angebote von Zeitungen in Deutschland. Im Jahr 2000 gab es nur 230, im Jahr 1995 erst fünf dererlei Angebote.

Discovery Networks International gibt am 2. November den Abschluss einer Vereinbarung mit der T-Online International AG bekannt, dank derer Nutzer über den eigenen PC und eine ADSL-Verbindung direkten Zugriff auf Programminhalte von Discovery erhalten. Bis zu zehn Dokumentationen können die Internet-Teilnehmer über das Portal „T-Online Vision“ unter dem Menüpunkt „Video on Demand“ abrufen. Die Abrechnung erfolge dann pro Film („Pay-per-Use-Basis“).

\subsection{Werbung / Marktanteile}

Nach Angaben des Zentralverbandes der deutschen Werbewirtschaft (ZAW) steigen die Investitionen in Werbung (Honorare, Kosten für Produktion und Verbreitung) im Jahr 2004 um 1,1 Prozent auf 29,2 Mrd. Euro. Damit wuchs monetär das Werbevolumen um 310 Mio. Euro. Im Jahr zuvor waren die Ausgaben für Werbung noch um 780 Mio. Euro gesunken. Der ZAW sieht daher die Rezession im deutschen Werbemarkt weitgehend beendet. Die Rückkehr zum Werbewachstum widerspräche der im Werbeabschwung immer wieder verbreiteten Position vom Verfall massenmedialer Werbung. Offenkundig sei Werbung in klassischen Medien für die marktkommunikationspolitischen Strategien der Unternehmen unverzichtbar. Die früher im Werbemarkt vorherrschende Stabilität sei indessen noch nicht eingeholt. So kommt 2004 der Anteil der Werbeinvestitionen am Bruttoinlandsprodukt (BIP) erst auf 1,34 Prozent gegenüber 1,64 Prozent im Jahr 2000.

Das Anzeigenaufkommen der Publikumszeitschriften steigt erstmals seit 2001 wieder. Nach Angaben des Verbandes Deutscher Zeitschriftenverleger (VDZ) buchten die Werbungtreibenden mit insgesamt 229.302 Anzeigenseiten 3,6 Prozent mehr als im Vorjahr. Zu den Gewinnern im Anzeigengeschäft gehören vor allem die monatlichen Frauenzeitschriften $(+7,0 \%)$, die Wirtschaftspresse (+ 5,3\%) und die aktuellen Zeitschriften und Magazine $(+3,2 \%)$. Die Wirtschaftstitel und die aktuellen Zeitschriften hingegen können die großen Verluste noch nicht ganz wettmachen. Besonders erfolgreich ist die Neugründung Audio Video Foto Bild mit einem Plus von 20 Prozent, im Segment der Fernsehzeitschriften trägt vor allem der Erfolg der neuen Springer-Zeitschrift „TV Digital“ zu dem Wachstum von zehn Prozent bei.

Angaben der Informationsgemeinschaft zur Feststellung der Verbreitung von Werbeträgern (IVW) zufolge beenden die Presseerzeugnisse das Jahr 2004 mit gemischter Auflagenbilanz. ${ }^{34}$ Die Gesamtauflage der Tageszeitungen und Zeitschriften sinkt im 
Tabelle: Werbeträger in Deutschland

\begin{tabular}{|c|c|c|c|c|c|c|}
\hline \multirow[t]{2}{*}{ Mediengruppe } & \multicolumn{2}{|c|}{ Anzahl } & \multirow{2}{*}{$\begin{array}{c}\text { Veränderung } \\
\text { in Prozent }\end{array}$} & \multicolumn{2}{|c|}{ Auflage } & \multirow{2}{*}{$\begin{array}{c}\text { Veränderung } \\
\text { in Prozent }\end{array}$} \\
\hline & 1999 & 2004 & & 1999 & 2004 & \\
\hline Tageszeitungen & 393 & 379 & $-3,6$ & 29,3 Mio. & 26,5 Mio. & $-9,6$ \\
\hline Wochenzeitungen & 24 & 26 & $+8,3$ & 2,2 Mio. & 2,1 Mio. & $-4,5$ \\
\hline Anzeigenblätter & 1311 & 1306 & $-0,4$ & 88,5 Mio. & 85,6 Mio. & $-3,3$ \\
\hline Publikumszeitschrif & ten 839 & 850 & $+1,3$ & 138,5 Mio. & 137,6 Mio. & $-0,6$ \\
\hline Fachzeitschriften & 1089 & 1064 & $-2,3$ & 26,3 Mio. & 23,5 Mio. & $-10,6$ \\
\hline Kundenzeitschriften & 88 & 85 & $-3,4$ & 63,0 Mio. & 51,1 Mio. & $-18,8$ \\
\hline $\begin{array}{l}\text { Telekommunikation } \\
\text { verzeichnisse }\end{array}$ & s- 155 & 242 & $+56,1$ & 36,0 Mio. & 37,0 Mio. & $+2,7$ \\
\hline $\begin{array}{l}\text { Massendrucksachen } \\
\text { Infopost }\end{array}$ & - & - & - & 6,4 Mrd. & 6,9 Mrd. & $+7,8$ \\
\hline $\begin{array}{l}\text { TV-Programme } \\
\text { bundesweit, landesw } \\
\text { regional u. lokal }\end{array}$ & reit, 107 & 156 & $+45,8$ & $\begin{array}{l}\text { 34,7 Mio. } \\
\text { angemeldete }\end{array}$ & $\begin{array}{l}\text { 36,4 Mio. } \\
\text { TV-Geräte }\end{array}$ & $+4,9$ \\
\hline $\begin{array}{l}\text { Hörfunkprogramme } \\
\text { bundesweit, landesw } \\
\text { regional u. lokal }\end{array}$ & eit, 236 & 331 & $+40,3$ & $\begin{array}{c}\text { 39,9 Mio. } \\
\text { angemeldete l }\end{array}$ & $\begin{array}{l}\text { 41,6 Mio. } \\
\text { Radiogeräte }\end{array}$ & $+4,3$ \\
\hline Online-Angebote & 142 & 398 & $+180,3$ & $\begin{array}{l}\text { 6,9 Mrd. } \\
\text { Seitenkor }\end{array}$ & $\begin{array}{l}\text { 68,1 Mrd. } \\
\text { ntakte }\end{array}$ & $+887,0$ \\
\hline Außenwerbung & 419645 & 403454 & $-3,9$ & - & - & \\
\hline Kino (Leinwände) & 4651 & 4870 & $+4,7$ & $\begin{array}{l}\text { 149,0 Mio. } \\
\text { Kinobesu }\end{array}$ & $\begin{array}{l}\text { 156,7 Mio. } \\
\text { ucher }\end{array}$ & $+5,2$ \\
\hline
\end{tabular}

Quelle: Zentralverband der deutschen Werbewirtschaft (ZAW)

4. Quartal 2004 im Vergleich zum Vorjahresquartal leicht. Die Tageszeitungen (einschließlich der aktuellen Sonntagsausgaben sowie Sonntagszeitungen) schließen das Jahr 2004 mit einer in der Gesamtentwicklung unveränderten Auflage ab; ihre verkaufte Auflage liegt im 4. Quartal 2004 mit 25,90 Mio. Stück gegenüber den 26,02 Mio. verkauften Exemplaren des vorangegangenen Vierteljahrs nahezu auf gleicher Höhe. Im Jahresvergleich (4/03: 26,39 Mio.) ergibt sich ein Auflagenrückgang von 0,49 Mio. Stück, die im Quartalsdurchschnitt pro Erscheinungstag weniger abgesetzt wurden. Die aktuelle Verkaufsauflage von 25,90 Mio. Exemplaren setzt sich zusammen aus 16,62 Mio. Abonnements, 8,27 Mio. Einzelverkäufen, 0,33 Mio. Bordexemplaren und 0,69 Mio. sonstigen Verkäufen.

Die Wochenzeitungen können im 4. Quartal 2004 eine verkaufte Auflage von 1,93 Mio. Stück verzeichnen; diese verteilt sich auf 1,57 Mio. Abonnements, 0,16 Mio. Einzelverkäufe, 0,04 Mio. Bordexemplare und 0,15 Mio. im sonstigen Verkauf abgesetzte Stücke; im Jahresvergleich zeigt sich allerdings ein marginaler Rückgang von 0,05 Mio. Exemplaren.

Die Auflagen der Publikumszeitschriften sind im Jahresvergleich leicht rückläufig. Insgesamt werden im Jahr 2004 123,64 Mio. Exemplare verkauft, die sich zusammensetzten aus 55,57 Mio. Abonnements, 49,54 Mio. Einzelverkäufen, 4,95 Mio. Lesezirkelstücken, 2,34 Mio. Bordexemplaren und 11,26 Mio. sonstigen Verkäufen. Im 4. Quartal des Vorjahres wurden insgesamt 125,37 Mio. Stück abgesetzt. Die durch- 
schnittlich im 4. Quartal 2004 pro Ausgabe verkaufte Auflage der deutschen Fachzeitschriften liegt bei 14,46 Mio. Exemplaren, die sich zusammensetzte aus 10,22 Mio. Abonnements, 0,21 Mio. Stück Einzelverkäufen sowie 4,01 Mio. sonstigen Verkäufen. Im 4 Quartal des Vorjahres waren es insgesamt 15,54 Mio. Exemplare. Auch die Kundenzeitschriften müssen gegenüber dem Vorjahr rückläufige Auflagen verzeichnen: Im 4. Quartal 2004 werden 51,09 Mio. Exemplare verbreitet (57,12 Mio. in 4/03), von denen 42,75 Mio. Stück verkauft wurden (43,79 Mio. in 4/03).

Nach der am 20. Juli veröffentlichten Media-Analyse 2004 Radio II (MA 2004 Radio II) ist unter den werbefreien, nationalen Sendern erneut der Deutschlandfunk Spitzenreiter, muss allerdings Verluste hinnehmen; er kommt auf 1,08 Mio. „Hörer gestern“ (MA 2004/I: 1,2 Mio.). RTL Radio erreicht 710.000 „Hörer gestern“ (+ 22.000), KlassikRadio 527.000 (- 46.000), Sunshine live 420.000 Hörer (+33.000) und Deutschlandradio Berlin zählt 277.000 „Hörer gestern“ (+12.000). Die drei meistgehörten Sender sind das private Mantelprogramm für den NRW-Lokalfunk Radio NRW mit 4,6 Mio. Hörern gestern (MA 2004/I: 4,4 Mio.), SWR 3 mit 3,3 Mio. Hörern gestern (MA 2004/I: 3,0 Mio.) und WDR 4 mit fast 3 Mio. Hörern gestern (MA 2004/I: 2,9 Mio.). EinsLive (WDR) kommt mit 2,8 Mio. Hörern gestern auf den vierten Platz (- 268.000 Hörer). Im Werbefunk erhöhte sich die Bruttoreichweite laut Arbeitsgemeinschaft Media-Analyse (AG.MA) um 1,6 Prozent auf 21 Mio. Kontakte pro Stunde. Größter Reichweitengewinner sind der schleswig-holsteinische Sender Delta Radio, der seine Bruttoreichweite (die Zahl der Hörer pro durchschnittlicher Stunde von Montag bis Samstag zwischen 6 und 18 Uhr) um 46,6 Prozent auf 85.000 Hörer pro Werbestunde erhöht. Bayern 4 Klassik steigert seine Reichweite um 41,2 Prozent auf 96.000 Hörer pro Werbestunde. Zu den Reichweitengewinnern gehören auch Radio 21, Bremen eins, 89.0 RTL, Berliner Rundfunk 91,4, Die Nene 107,7, Energy Sachsen, Ostseewelle Mecklenburg-Vorpommern und SWR 1 in Rheinland-Pfalz. In der MA 2004/II wurde mithilfe von 60.000 Telefoninterviews die Nutzung von 331 Sendern (acht mehr als in der MA 2004/I) erhoben, davon waren 62 öffentlich-rechtlich, 197 privat und 72 sonstige Sender. Zu den sonstigen Sendern gehören die nichtkommerziellen Radios, Offene Kanäle und ausländische Sender.

Die ARD kann RTL in der Rolle des so genannten Marktführers unter den Fernsehsendern im Jahr 2004 ablösen. Nach Zahlen der Gesellschaft für Konsumforschung (GfK) erzielt die ARD bezogen auf alle Zuschauer einen Marktanteil von 14 Prozent ($0,1 \%$ im Vergleich zum Vorjahr). Den zweiten Platz belegten gemeinsam die sieben Dritten Programme der ARD-Anstalten, die bundesweit auf zusammen 13,9 Prozent Marktanteil kommen (+ 0,3 \%), gefolgt von ZDF und RTL, die jeweils 13,8 Prozent $(\mathrm{ZDF}+0,4 \%, \mathrm{RTL}-1,1 \%)$ erzielen. Es folgen Sat.1 mit 10,3 Prozent (+0,1\%) und ProSieben mit 7 Prozent (+/- $0 \%$ ). Bei den kleineren Programmen erzielte RTL II 4,9 Prozent (+ 0,2 \%), Kabel1 4,0 Prozent (- 0,1 \%), Vox 3,7 Prozent (+ 0,3 \%). Relativ unverändert zum Vorjahr bleiben Super RTL mit 2,7 Prozent, der Kinderkanal Kika mit 1,2 Prozent, DSF mit 1,1 Prozent, 3sat mit 1 Prozent, Eurosport mit 0,9 Prozent, N-TV und Phoenix mit jeweils 0,5 Prozent, MTV, NTV, N 24, Arte und VIVA mit jeweils 0,4 Prozent, Tele 5, MTV 2 und VIVA PLUS mit jeweils 0,3 Prozent sowie 9Live mit 0,2 Prozent. XXP (getragen von Spiegel TV und der 1988 von Alexander Kluge gegründeten Development Company for Television Programs, DCTP) erzielt einen Marktanteil von 0,1 Prozent. Innerhalb ihrer eigenen Sendegebiete ist das Fernsehen der Dreiländeranstalt MDR mit 9,4 Prozent am erfolgreichsten und liegt vor dem Fernsehen der Vierländeranstalt NDR (8,6 \%). Schlusslicht ist das HR Fernsehen mit 5,8 Prozent Marktanteil im eigenen Sendegebiet. Fasst man die auf ARD, ZDF, die Dritten Programme und 3sat entfallenden Marktanteile zusammen und vergleicht sie mit dem ent- 
sprechenden Summenwert für RTL, Sat.1, ProSieben, RTL II, VOX und Kabel 1, so sind bei den unter 50-Jährigen sowohl in Ost- als auch in Westdeutschland die Privatsender (61,2 \% vs. 58,2 \%) die beliebteren Sender. Die über 50-Jährigen hingegen bevorzugen die öffentlich-rechtlichen Programme.

Unter den 10 meistgesehenen Fernsehsendungen 2004 sind die Spitzenreiter sechs Fußballübertragungen der von ARD und ZDF ausgestrahlten Europameisterschaft in Portugal. Die übrigen vier Top-10-Sendungen sind allesamt Ausgaben der ZDF-Sendung „Wetten, dass ...?“.

\section{Mediennutzung}

Im Jahr 2004 beträgt die Reichweite der Tageszeitungen der Media Analyse zufolge 75,5 Prozent, drei Viertel der Bevölkerung über 14 Jahren lesen damit Zeitungen. Das bedeutet einen geringen Rückgang gegenüber 2003 von 0,3 Prozent, wobei die Reichweite der Tageszeitungen in den alten Bundesländern ein Minus von nur 0,3 Prozent aufweist, während sie in den neuen Bundesländern um 1,4 Prozent sinkt. Die regionalen Abonnementszeitungen liegen mit einer Reichweite von 64,2 Prozent geringfügig unter dem Wert des Vorjahres (64,8 \%). Auch die Kaufzeitungen liegen im Vergleich zu 2003 mit jetzt 22,4 Prozent (2003: 22,7 \%) fast gleich. Die überregionalen Tageszeitungen kommen auf eine Reichweite von 5,5 Prozent. Insgesamt ist die Altersstruktur der Zeitungsleser relativ konstant: Der Zeitungskonsum nimmt mit dem Alter deutlich zu. Die höchsten Reichweiten erzielen die Tageszeitungen bei den 60- bis 69-Jährigen mit 84,8 Prozent. Von den 14- bis 19-Jährigen werden hingegen nur 51,8 Prozent erreicht, bei den jungen Erwachsenen zwischen 20 und 29 Jahren greifen 61,5 Prozent zur Zeitung.

Die Radionutzung nimmt nach der Media-Analyse 2004 Radio II (MA 2004 Radio II) weiter zu: An jedem Werktag schalten 52,54 Mio. Personen ab 14 Jahren (81,2 \%) mindestens ein Radioprogramm ein; das sind 400.000 mehr als bei der vorangegangenen Media-Analyse. Die Verweildauer der Radiohörer erhöht sich minimal von 258 (MA 2004/I) auf 259 Minuten täglich, wobei Männer mit 217 Minuten am Tag länger Radio hören als Frauen mit nur 204 Minuten. Nach Angaben der ARD schalteten täglich fast 35 Mio. Hörer täglich mindestens ein Radioprogramm der ARD ein (MA 2004/I: 34,4, Mio.). Alle Privatsender zusammen haben zwischen Montag und Freitag 29,4 Mio. Hörer täglich (MA 2004/I: 28,2 Mio.). Nach Angaben des Werbevermarkters der ARD, ARD Sales \& Services (AS\&S), ist die tägliche Radionutzung vor allem bei den „konsumkräftigen Personen zwischen 30 und 59 Jahren “ überdurchschnittlich hoch. 85,8 Prozent dieser Bevölkerungsgruppe höre täglich Radio, und dies länger als vier Stunden (Verweildauer: 281 Minuten). Auch die jungen Nutzer hören wieder länger Radio: 77 Prozent der 14- bis 29-Jährigen schalteten das Radio täglich ein und hörten durchschnittlich 165 Minuten zu - 14 Minuten länger als noch bei der jüngsten MA.

Immer mehr Haushalte empfangen ihr Fernseh- und Radioprogramm auf digitalem Weg: Während Ende 2001 erst 2,04 Mio. Haushalte über einen digitalen Kabel-, Satelliten- oder Antennenanschluss verfügten, sind es Ende 2004 bereits 5,27 Mio. Haushalte, allein 870.000 davon empfangen DVB-T in denjenigen Gebieten, in denen das analoge terrestrische Signal abgeschaltet wurde. Nach Angaben der ARD-Medienforschung beträgt die durchschnittliche Fernsehdauer pro Tag im Jahr 2004210 Minuten, das sind 7 Minuten mehr als im Vorjahr. Der Mehrkonsum verdankt sich vor allem den erwachsenen Zuschauern über 14 Jahren, die 225 Minuten täglich schauen, während die Kinder von 3-13 Jahren gegenüber 2003 unverändert täglich 93 Minuten vor dem Fernseher verbringen. Nach wie vor wird in Ostdeutschland mit 238 Minuten insgesamt 35 Minuten 
länger ferngesehen als in Westdeutschland mit 203 Minuten. Die Tagesreichweite des Fernsehens liegt 2004 bei 74 Prozent (Erwachsene: 76 \%, Kinder: $61 \%$ ).

Nach Angaben der „Online-Studie 2004“ von ARD und ZDF, die am 6. September vorgestellt wird, sinkt die durchschnittliche Nutzungszeit im Internet 2004 im Vergleich zum Vorjahr um zwei Minuten auf 43 Minuten täglich. 35,7 Mio. Erwachsene, das entspricht 55,3 Prozent der Bevölkerung ab 14 Jahren, sind online, doppelt so viele wie im Jahr 2000. Besonders hohe Zuwachsraten gibt es in den neuen Bundesländern: Mit einer Internetverbreitung von 52,3 Prozent hat sich die Nutzung dort fast an das Niveau der alten Bundesländer (56,1 \%) angeglichen. Nach wie vor wird das Internet am häufigsten von 14- bis 19-Jährigen (94,7 \%) und Berufstätigen (73,4 \%) genutzt, am wenigsten von Bürgern, die älter als 60 Jahre (14,5\%) oder nicht berufstätig sind $(22,9 \%)$. Die Zahlungsbereitschaft für kostenpflichtige Angebote sinkt der Umfrage zufolge von 27 Prozent in 2001 auf nur noch 18 Prozent 2004. Für den größten Teil der Internetnutzer stehe die Kommunikation und die Informationsbeschaffung im Vordergrund. Deutlich zugelegt haben der Zugang zu Märkten und Produkten sowie zu internetspezifischen Serviceleistungen. Der Trend bei den nachgefragten Inhalten gehe eindeutig zu „Spezialisten für bestimmte Themenbereiche“, etwa Nachrichtenanbieter und Ratgeber- sowie Verbraucherseiten, wobei vor allem jene Angebote nachgefragt würden, die schon in Form von Presse, Fernsehen und Hörfunk hohe Akzeptanz aufwiesen. Unterhaltungsangebote spielten weiterhin eine geringe Rolle.

Daten der Forschungsgruppe Wahlen zufolge verfügten im vierten Quartal 2004 fast zwei Drittel der Deutschen über einen Zugang zum Internet, ein Plus von 4 Prozent verglichen mit dem Vorjahresquartal. Nahezu unverändert rufen 30 Prozent der Internetnutzer regelmäßig Politiknachrichten ab, Börsen- und Wirtschaftsnachrichten werden aber nur noch von 32 Prozent der Nutzer (- 4 \%) angeklickt. 54 Prozent der Nutzer nutzen das Internet zum Einkaufen (+ $5 \%$ ), 57 Prozent zum Preisvergleich (+ $1 \%$ ). Im Gegensatz zur Onlinestudie von ARD und ZDF kommt die Forschungsgruppe Wahlen zum Ergebnis, dass sich die Nutzungsintensität des Netzes kaum verändert habe: 36 Prozent der Deutschen mit Internetzugang verbrächten in der Woche bis zu zwei Stunden im World Wide Web, weitere 37 Prozent seien zwischen drei und zehn Stunden online. 16 Prozent verweilten pro Woche mehr als zehn Stunden im Internet. Der prozentuale Anteil der Männer, die mehr als zehn Stunden im Internet verbrächten, sei mehr als doppelt so hoch wie der der Frauen (22\% gegenüber $10 \%)$. Bei den über 60-Jährigen stieg der Anteil der Internet-Nutzer um sechs Prozentpunkte auf 30 Prozent. Die formale Bildung sei nach wie vor ausschlaggebend dafür, ob jemand einen Internet- Zugang besitzt: Mehr als vier Fünftel der Deutschen mit Hochschulreife verfügten über einen Zugang zum Internet, aber nur ein Fünftel der Hauptschüler ohne Lehre.

Der Fernsehkonsum nimmt von 221 Minuten im ersten Halbjahr 2003 auf 230 Minuten im ersten Halbjahr 2004 zu, die Radionutzung bleibt mit 196 Minuten täglich stabil.

Ende 2004 sind 42.170.910 Rundfunkempfangsgeräte (+ 542.633 gegenüber dem Vorjahr) bei der Gebühreneinzugszentrale (GEZ) angemeldet, 2.047.849 Teilnehmer haben nur ein Radiogerät angemeldet, 3.492.342 Geräte sind gebührenbefreit.

Quellen: Funkkorrespondenz, epd medien, Media Perspektiven, DocuWatch Digitales Fernsehen, Presseinformationen der Sender, Landesmedienanstalten und der GEZ, eigene Recherchen 